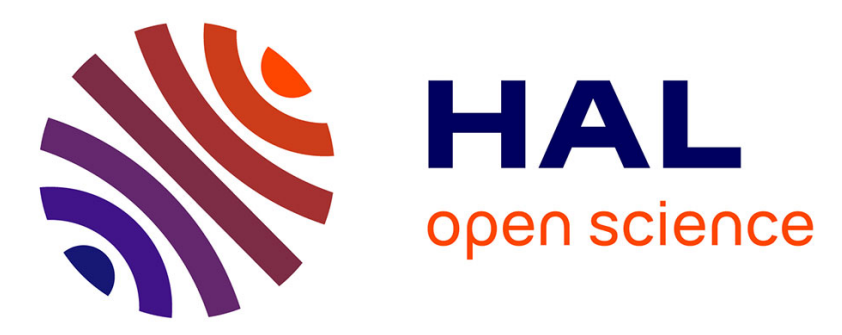

\title{
Spin-transport Mediated Single-shot All-optical Magnetization Switching of Metallic Films
}

Satoshi Iihama, Quentin Remy, Junta Igarashi, Grégory Malinowski, Michel Hehn, Stéphane Mangin

\section{- To cite this version:}

Satoshi Iihama, Quentin Remy, Junta Igarashi, Grégory Malinowski, Michel Hehn, et al.. Spintransport Mediated Single-shot All-optical Magnetization Switching of Metallic Films. Journal of the Physical Society of Japan, 2021, 90, pp.081009. 10.7566/JPSJ.90.081009 hal-03433380

\section{HAL Id: hal-03433380 \\ https://hal.univ-lorraine.fr/hal-03433380}

Submitted on 18 Nov 2021

HAL is a multi-disciplinary open access archive for the deposit and dissemination of scientific research documents, whether they are published or not. The documents may come from teaching and research institutions in France or abroad, or from public or private research centers.
L'archive ouverte pluridisciplinaire HAL, est destinée au dépôt et à la diffusion de documents scientifiques de niveau recherche, publiés ou non, émanant des établissements d'enseignement et de recherche français ou étrangers, des laboratoires publics ou privés. 


\title{
Spin-transport Mediated Single-shot All-optical Magnetization Switching of Metallic Films
}

\author{
Satoshi Iihama ${ }^{1,4,5 *}$, Quentin Remy², Junta Igarashi ${ }^{3}$, Grégory Malinowski², \\ Michel Hehn'2, and Stéphane Mangin ${ }^{2}$ \\ ${ }^{1}$ Frontier Research Institute for Interdisciplinary Sciences, Tohoku University, Sendai 980-8578, Japan \\ ${ }^{2}$ Université de Lorraine, CNRS, Institut Jean Lamour, F-54000, Nancy, France \\ ${ }^{3}$ Laboratory for Nanoelectronics and Spintronics, Research Institute of Electrical Communication, Tohoku University, \\ Sendai 980-8577, Japan \\ ${ }^{4}$ Center for Spintronics Research Network, Tohoku University, Sendai 980-8577, Japan \\ ${ }^{5}$ WPI Advanced Institute for Materials Research (AIMR), Tohoku University, Sendai 980-8577, Japan \\ (Received January 5, 2021; accepted May 20, 2021; published online June 30, 2021)
}

\begin{abstract}
Ultrafast control of thin film nanomagnets using femtosecond laser pulses has attracted considerable attention for future ultrafast and energy-efficient photo-spintronics storage/memory devices. Single femtosecond laser pulse magnetization switching without an applied external magnetic field has been recently observed on various metallic thin films. Herein, a recent understanding of the physical mechanism behind single-shot all-optical magnetization switching and perspectives are described.
\end{abstract}

\section{Introduction}

Ultrafast manipulation of thin film magnetization using femtosecond (fs) laser pulses has been investigated in the past decades. The use of ultrashort laser pulses enables the study/ manipulation of ultrafast electron and spin dynamics. ${ }^{1-7)}$ Because ultrafast laser heating in metals induces significant fluctuations of magnetic moments, the ultrafast magnetization dynamics of ferromagnets (FMs) cannot be described by a simple macrospin model. In 1996, ultrafast quenching of magnetization, known as ultrafast demagnetization, within sub-picosecond (ps) time scale in a nickel thin film was first demonstrated. ${ }^{1)}$ Subsequently, many associated physical phenomena such as ultrafast hot electron/spin transport ${ }^{8-12)}$ and terahertz emission ${ }^{13,14}$ have been observed. The superdiffusive transport of photo-excited carriers within the thermalization time has attracted significant attention. ${ }^{15-19)}$ Ultrafast spin transport can be induced by a fs laser pulse focused on a metallic multilayers containing FMs. Spin transport is triggered by the ultrafast quenching of magnetic moments in FMs owing to the conservation of angular momentum. Furthermore, intersite spin transfer in ferromagnetic alloys as well as the transfer of angular momentum between two ferromagnetic layers separated by a nonmagnetic spacer layer have been demonstrated. ${ }^{20-27)}$

Simultaneously, magnetization switching induced by irradiation with a fs laser pulse without an applied external magnetic field, generally known as all-optical switching (AOS), has been demonstrated for various magnetic materials; hence, it can potentially be applied in future ultrafast optically enabled spintronics devices. ${ }^{28)}$ AOS was first demonstrated in a ferrimagnetic amorphous rare-earth (RE) transition-metal (TM) alloy, i.e., GdFeCo (GFC). ${ }^{29)}$ In this alloy, the magnetization of the $\mathrm{Gd}$ and FeCo sublattices are coupled antiferromagnetically. AOS in GFC was initially discussed in terms of the effect of circularly polarized light due to the inverse Faraday effect. Subsequently, it was demonstrated that the magnetic moments of the two sublattices can be reversed by the irradiation of a linearly polarized single fs laser pulse $\mathrm{s}^{30,31)}$ as schematically shown in Fig. 1(a). The time-, space-, and element-resolved ultrafast (a)

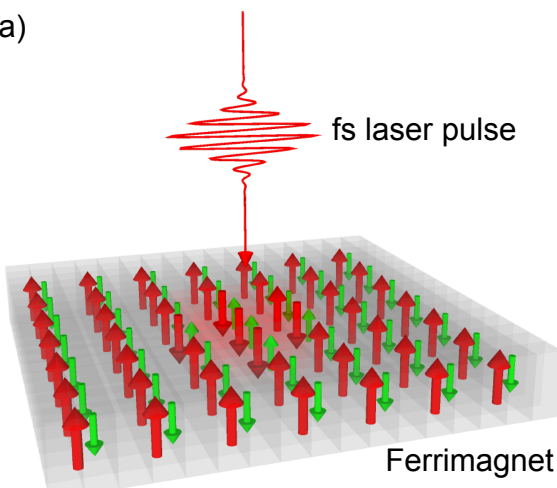

(b)

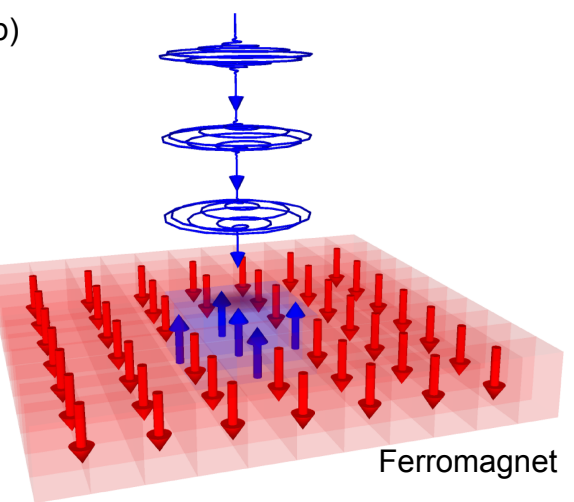

Fig. 1. (Color online) (a) Schematic illustration of single-shot AOS in ferrimagnet. Magnetic moments of two sublattices can be reversed by irradiation of single fs laser pulse. (b) Schematic illustration of AOS in ferromagnet. Magnetization direction can be reversed owing to the helicity of the laser. This switching can be typically achieved by multiple laser pulses.

magnetization dynamics in GFC have been investigated intensively. ${ }^{30,32-39)}$ Single-shot AOS of GFC is mediated by the transient ferromagnetic state owing to the angular momentum transfer between the two sublattices. ${ }^{40,41)}$ Although several studies have demonstrated single-shot AOS in Tb-based RE-TM system such as TbFeCo alloy, ${ }^{42)}$ [Tb/Co] multilayer ${ }^{43)}$ and ferrimagnetic Heusler alloy $\mathrm{Mn}_{2} \mathrm{Ru}_{x} \mathrm{Ga}^{44,45)}$ single-shot AOS has been primarily inves- 
tigated using Gd-based materials including synthetic ferrimagnets such as $\mathrm{Co} / \mathrm{Gd}^{46-48)}$

Whereas many studies on single-shot AOS have been performed on ferrimagnetic thin films, single-shot AOS for FMs has been considered difficult. In contrast to the fact that AOS can be induced by linearly polarized laser pulses in the GFC ferrimagnet, AOS in FMs has only been observed by using the helicity of the laser pulse ${ }^{49-52)}$ as schematically shown in Fig. 1(b). However, the mechanism of magnetization switching for FMs is still debated. In all-optical helicity dependent switching (AO-HDS), the magnetization of various types of magnetic materials can be reversed by sweeping the laser pulses or after irradiation of many laser pulses. ${ }^{29,49,52-60)}$ Accumulative magnetization switching due to magnetic circular dichroism ${ }^{61,62)}$ and/or light helicity-induced magnetization $^{63,64)}$ might be a contributing factor. In addition, helicity-dependent laser-induced domain wall motion could also play an important role. ${ }^{65,66)}$ Another mechanism of AOHDS for FMs is that magnetization is switched due to the stabilization of the magnetic domain when the size of the domain is comparable to that of the laser spot. ${ }^{56,67)}$ Although AOS even in FMs has been realized by using various methods, single-shot AOS that has been demonstrated in ferrimagnets will be promising for the ultrafast and energyefficient magnetization switching. Single-shot AOS in FMs was initially realized using ferromagnetic $\mathrm{Co} / \mathrm{Pt}$ multilayers exchange-coupled with the GFC layer. ${ }^{68)}$ Subsequently, single-shot AOS of a FM layer was achieved by utilizing the spin transport in a spin-valve structure where two magnetic layers are magnetically decoupled. ${ }^{69}$

Furthermore, our recent results demonstrated that energyefficient single-shot AOS of FMs can be obtained by increasing the amount of spin-current using a FM/nonmagnetic metal/GFC spin-valve structure. ${ }^{70)}$ From the experiments and calculations pertaining to the ordering temperature of the FMs in a spin-valve, the mechanism of single-shot AOS of the FMs can be qualitatively explained by the combined effects of ultrafast quenching of magnetization and the transfer of angular momentum from Gd. ${ }^{71}$ )

In this review, we first describe the AOS in ferrimagnetic GFC and the mechanism behind single-shot AOS. Next, spin-transport following fs laser excitation in the metallic multilayer stack is introduced. Next, we describe spintransport-mediated single-shot AOS and the energy required for magnetization switching of FMs. The physical mechanism behind single-shot magnetization switching of FMs is discussed. Finally, the perspectives for single-shot AOS are discussed in the light of numerous experimental results.

\section{AOS in a GdFeCo Alloy Ferrimagnet}

The GFC alloy is an amorphous RE-TM ferrimagnet, and the magnetization of the RE and TM sublattices are opposite because of the negative exchange coupling between the two elements as schematically shown in Fig. 2(a). The first observation of AOS was demonstrated in GFC. ${ }^{29)}$ Figure 2(b) shows the magnetization reversal by the irradiation of a $40 \mathrm{fs}$ circularly polarized single laser pulse. The magnetization reversal depended on the helicity of the laser pulse [Fig. 2(b) top]. Because the magnetization can be reversed by the helicity of laser pulses without the use of an external magnetic field, all-optical magnetic recording [Fig. 2(b) (a)

(b)
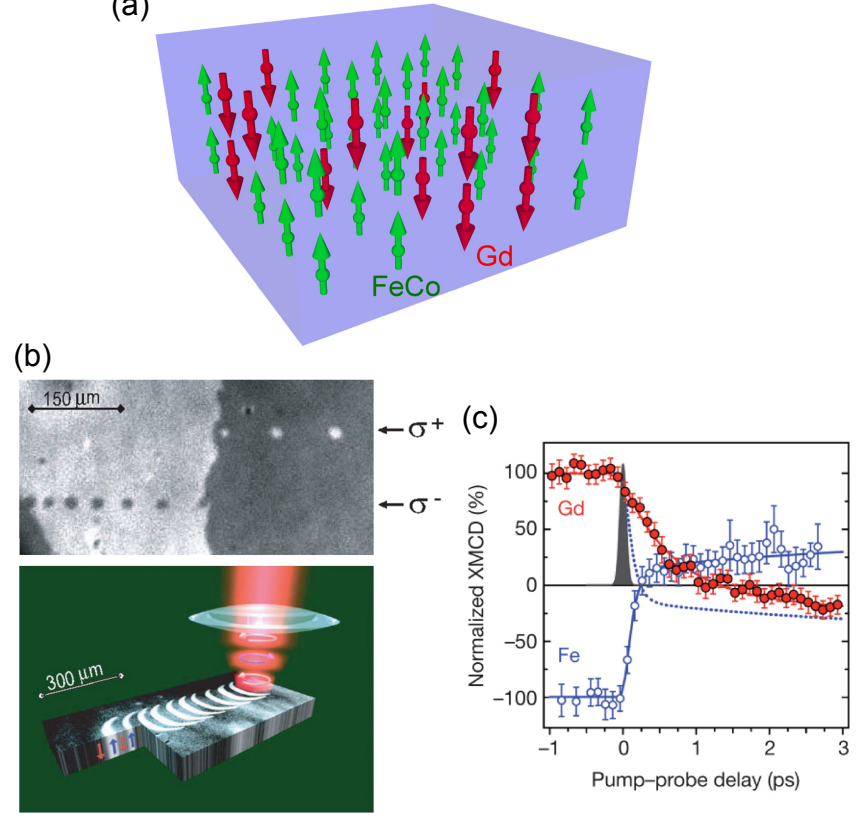

(d) a

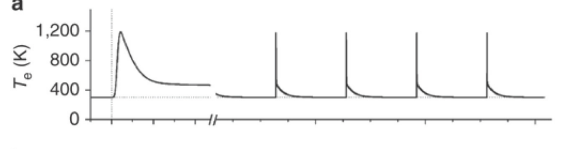

b

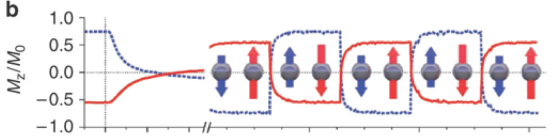

c

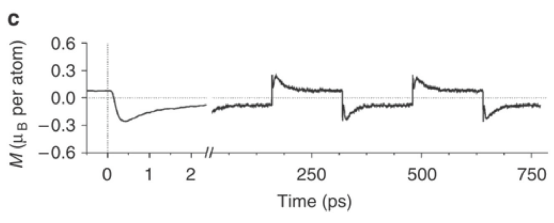

Fig. 2. (Color online) (a) Schematic illustration of GFC ferrimagnet. Gd and FeCo have opposite magnetic moments due to antiferromagnetic exchange coupling. (b) All-optical magnetization switching demonstrated for GFC film. Reprinted with permission from Stanciu et al. ${ }^{29)}$ (C) 2007 American Physical Society. (c) Transient ferromagnetic states observed in GFC by time-resolved X-ray magnetic circular dichroism experiment. Reprinted with permission from Radu et al. ${ }^{30)}$ (C) 2011 Nature Publishing Group. (d) Atomistic spin dynamics simulation results of all-optical magnetization switching. Each magnetic moment (middle) and total magnetic moment (bottom) dynamics induced by ultrafast laser-heating (top). Reprinted with permission from Ostler et al. ${ }^{31)}$ (C) 2012 Nature Publishing Group.

bottom] was proposed. The mechanism of the AOS was first discussed based on an effective magnetic field generated by the inverse Faraday effect. ${ }^{29,33,34)}$ Subsequently, AO-HDS in GFC was quantitatively explained based on magnetic circular dichroism, ${ }^{72)}$ i.e., light absorption is different with the light helicity. Simultaneously, it was demonstrated that the magnetization of GFC was reversed by single linearly polarized fs laser pulses. This result indicated that the mechanism of single-shot AOS is of purely thermal origin. Time-resolved X-ray magnetic circular dichroism measurements were performed to study the element-resolved magnetization dynamics. ${ }^{30}$ ) Figure 2(c) shows the time- and element-resolved $\mathrm{Gd} / \mathrm{Fe}$ magnetization dynamics. A transient ferromagnetic-like state at approximately $1 \mathrm{ps}$ was observed. The different demagnetization rates for $\mathrm{Gd}$ and $3 d$ transition 
metals such as Fe was originally explained by the microscopic three temperature model (M3TM), ${ }^{73}$ in which the demagnetization is caused by spin-flip scattering mediated by electron-phonon scattering. In the M3TM, the demagnetization rate $\tau_{\mathrm{M}}^{-1}$ is characterized by the spin-flip probability $a_{\text {sf }}$, magnetic moment $\mu$ and the Curie temperature $T_{\mathrm{C}}$ as follows: ${ }^{73)}$

$$
\tau_{\mathrm{M}}^{-1} \propto a_{\mathrm{sf}} T_{\mathrm{C}}^{2} / \mu
$$

Therefore, the large magnetic moment and low $T_{\mathrm{C}}$ of $\mathrm{Gd}$ yielded a lower demagnetization rate.

Thermally induced single-shot AOS was theoretically investigated using atomistic spin dynamics simulations. ${ }^{30,31)}$ The dynamics of spin $S_{i}$ at each localized lattice was calculated using the Landau-Lifshitz-Gilbert (LLG) equation as follows:

$$
\frac{\partial \boldsymbol{S}_{i}}{\partial t}=-\frac{\gamma}{\left(1+\alpha_{i}^{2}\right) \mu_{i}}\left[\boldsymbol{S}_{i} \times \boldsymbol{H}_{i}+\alpha_{i} \boldsymbol{S}_{i} \times\left(\boldsymbol{S}_{i} \times \boldsymbol{H}_{i}\right)\right],
$$

where $\gamma, \mu_{i}$, and $\alpha_{i}$ are gyromagnetic ratio, magnetic moment, and damping parameter, respectively. $\boldsymbol{H}_{i}\left(=-\partial E_{i} / \partial \boldsymbol{S}_{i}\right)$ expresses an effective magnetic field considering the anisotropy energy and exchange coupling energy, with

$$
E_{i}=-\sum_{n n} J_{i j} S_{i} \cdot S_{j}-K S_{i, z}^{2}
$$

where the summation considers the nearest-neighbor interaction. $J_{i j}$ and $K$ are the exchange coupling constant and the uniaxial anisotropy constant, respectively. A stochastic Langevin field was included in $\boldsymbol{H}_{i}$ to consider the effect of the laser heating. The temperature was considered as the electronic temperature, which can be calculated using a twotemperature model (TTM). Figure 2(d) shows computed thermally driven AOS, where five Gaussian laser pulses were irradiated on GFC. Toggle-type switching of each magnetic moment was demonstrated in this calculation. Stochastic atomistic spin dynamics simulations on various model systems have been reported and ultrafast thermally induced magnetization switching has been predicted theoretically. ${ }^{41,74-77)}$ Another type of numerical description can be obtained using the Landau-Lifshitz-Bloch equation. ${ }^{78)}$ Magnetization switching in RE-TM ferrimagnets has been reproduced theoretically using the Landau-Lifshitz-Bloch equation. ${ }^{79)}$ In addition, theoretical models based on microscopic theories exist for single-shot AOS in ferrimagnets. ${ }^{80-87)}$ In the microscopic model, exchange scattering of electron spins in the sublattices was considered to reproduce the magnetization switching in the ferrimagnet. The scattering of electron spins of two sublattices through intra-atomic exchange coupling such as $s-d$ exchange has been theoretically implemented to reproduce single-shot AOS in ferrimagnets. ${ }^{84-87)}$ Mentink et al. developed a simple model to explain the thermally induced magnetization reversal. ${ }^{40,88)}$ Figure 3(a) shows a schematic illustration of the spin angular momentum flow in the model. The spin-angular momenta of the two sublattice, $S_{1}$ and $S_{2}$ are described as follows:

$$
\begin{aligned}
& \frac{d S_{1}}{d t}=\lambda_{\mathrm{e}}\left(H_{1}-H_{2}\right)+\lambda_{1} H_{1}, \\
& \frac{d S_{2}}{d t}=-\lambda_{\mathrm{e}}\left(H_{1}-H_{2}\right)+\lambda_{2} H_{2},
\end{aligned}
$$

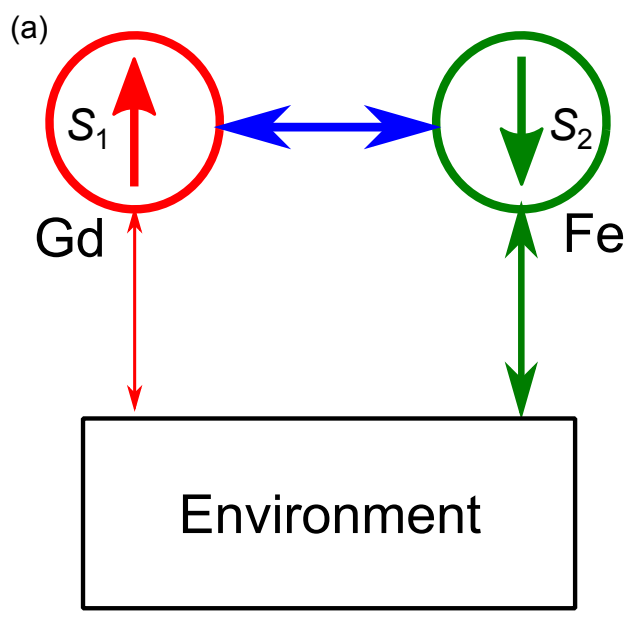

(b)

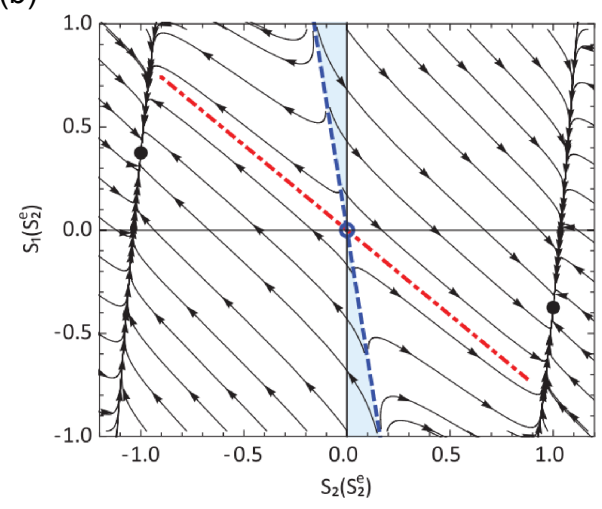

Fig. 3. (Color online) (a) Schematic illustration of path for spin-angular momentum flow in model introduced by Mentink et al. ${ }^{40)}$ Two arrows $S_{1}, S_{2}$ represent spin direction of two sublattices. Double sided arrows indicate the coupling. (b) Time evolution of $S_{1}$ plotted as a function of $S_{2}$ calculated using the model. Figure from Mentink et al. (C) 2012 Authors). ${ }^{40)}$

where the first and second terms on the right-hand side represent the transfer of angular momentum of each sublattice due to exchange scattering of electron spins and transfer of angular momentum to the environment. $\lambda_{\mathrm{e}}$ and $\lambda_{i}(i \in\{1,2\})$ represent the coupling strength of the two sublattices and the coupling between each sublattices with the environment, respectively. $H_{i}$ is the effective magnetic field, which can be calculated by the magnetic energy $W$ using functional derivative: $H_{i}=-\delta W / \delta S_{i}$. W is expressed as follows: ${ }^{40,89)}$

$$
W=\int d \boldsymbol{r}\left[f_{1}\left(S_{1}^{2}\right)+f_{2}\left(S_{2}^{2}\right)+f_{\mathrm{e}}\left(S_{1} S_{2}\right)\right] .
$$

Here, $f_{i}$ is the energy for each sublattice and is obtained using the Landau theory, where $f_{i}$ has a minimum at the spontaneous spin angular momentum $\left|\overline{S_{l}}\right|$ at room temperature below $T_{\mathrm{C}}$ for ferromagnets, i.e., $f_{i} \propto\left(S_{i}^{2}-{\overline{S_{l}}}^{2}\right)^{2} . f_{\mathrm{e}}$ is the exchange coupling energy which can be expressed as, $f_{\mathrm{e}}=-J_{\mathrm{e}} S_{1} S_{2}$. $\lambda_{i}$ can be described by the longitudinal relaxation time in the Bloch equation and therefore can be expressed as follows: ${ }^{90)}$

$$
\lambda_{i} \propto \frac{\gamma_{i} \alpha_{i} k_{\mathrm{B}} T}{\mu_{i}} .
$$

Thus, a larger damping parameter and a smaller magnetic moment increase the coupling to the environment. Here, we assume that $S_{1}$ and $S_{2}$ correspond to spins of $\mathrm{Gd}$ and $\mathrm{Fe}$ in the 
two sublattices, consequently, $S_{2}(>0)$ demagnetizes faster than $S_{1}(<0)$ and reaches 0 due to a large $\lambda_{i}$. Menink et al. calculated the time-evolution of $S_{1}$ and $S_{2}$ at constant temperature using Eqs. (3) and (4). Figure 3(b) shows timeevolution of $S_{1}$ plotted as a function of $S_{2}$ with various initial $S_{1}$ and $S_{2}$. In this calculation, $\lambda_{\mathrm{e}}$ is assumed to be larger than $\lambda_{i}$. If the first terms in the right-hand side of Eqs. (3), (4) are dominating, the relation $d S_{1} / d t=-d S_{2} / d t$ is obtained [shown as the red dotted line in Fig. 3(b)]. The two thick dots indicate equilibrium values. The blue shaded area represents the initial conditions from which the exchange of angular momentum will proceed to reversal. This condition indicates that the magnetic moment of one sublattice has to be quenched while the magnetic moment of another sublattice should still be finite. In addition to the above condition for the spin reversal, this model also yielded that exchange scattering of electron spin in an anti-parallel spin configuration increased the speed of demagnetization for both sublattices. ${ }^{40)}$

Based on the experimental studies and the theoretical considerations mentioned above, the significant difference in the demagnetization time and transfer of angular momentum between two sublattices are essential for realizing the singleshot AOS in GFC. Another important mechanism is the dissipation-less dynamics of the angular momentum during the demagnetization and switching processes. Wienholdt et al. calculated orbital-resolved atomistic LLG simulation with changing the damping parameter $\left[\alpha_{i}\right.$ in Eq. (1)] artificially. ${ }^{41)}$ It was theoretically demonstrated that the transient ferromagnetic states disappeared when higher values of damping were applied in the LLG calculation. Because the damping parameter reflects the angular momentum dissipation to the environment, it suppresses the exchange of angular momentum between two sublattices.

\section{Ultrafast Spin-transport in Metallic Multilayer Stacks}

When fs laser pulses are irradiated on a metallic structure, the strong absorption of photons results in a significant increase in the electron temperature. Consequently, heat flow is induced by the strong electron temperature gradient in the metallic structure. The electron and phonon temperature dynamics are generally described using the TTM, ${ }^{16,91-94)}$ where electron-phonon coupling, electronic heat diffusion, and the light absorption profile are considered. Simultaneously, spin-transport can be induced when fs laser pulses are irradiated on metallic multilayer stacks, including magnetic layers, as schematically shown in Fig. 4(a). It was theoretically predicted that ultrafast demagnetization is partially caused by the superdiffusive spin-transport in metallic films ${ }^{18)}$ in which the spin angular momentum is transferred from FM to a nonmagnet. The first experimental observation of the ultrafast spin-transport in a metallic film was reported using $\mathrm{Fe} / \mathrm{Au}$ by Melnikov et al. ${ }^{8)}$ In the first observation, a $35 \mathrm{fs}$ pump laser pulse was irradiated from the $\mathrm{Fe}$ layer side and a spin accumulation signal in $\mathrm{Au}$ was detected by using second harmonic generation on the opposite side of the Au layer. The spin accumulation dynamics obtained in Au are shown in Fig. 4(b). The spinpolarized carrier velocity evaluated from the shift of the peak for different $\mathrm{Au}$ thicknesses was comparable to the calculated velocity of the ballistic transport for the photo-excited carrier. ${ }^{8)}$ Front-side pump/back-side probe technique has been used to detect the ultrafast spin-transport in several studies. ${ }^{10-12,95)}$ In the experiment by Choi et al., a 0.8 ps laser pulse induced spin accumulation in a thick $\mathrm{Cu}$ layer in FM/ $\mathrm{Cu}$ samples. The spin accumulation in $\mathrm{Cu}$ was explained by the spin-current generation due to the ultrafast demagnetization in the FM layer. ${ }^{10)}$ If one assumes that spin-transport is described by a diffusive equation, then the spin accumulation in the nonmagnetic layer can be described as follows: ${ }^{96)}$

$$
\frac{\partial \mu_{\mathrm{s}}(z, t)}{\partial t}=D \frac{\partial^{2} \mu_{\mathrm{s}}(z, t)}{\partial z^{2}}-\frac{\mu_{\mathrm{s}}(z, t)}{\tau_{\mathrm{s}}}+Q_{\mathrm{s}}(t),
$$

where, $\mu_{\mathrm{s}}, D$, and $\tau_{\mathrm{s}}$ are the spin chemical potential, diffusion constant, and spin lifetime, respectively; $z$ is the coordinate along the thickness direction; $Q_{\mathrm{s}}(t)$ is a source term at the $\mathrm{FM} /$ nonmagnet interface owing to ultrafast demagnetization, which can be expressed as

$$
Q_{\mathrm{s}}(t) \propto-\frac{d S_{\mathrm{FM}}(t)}{d t},
$$

where, $S_{\mathrm{FM}}$ is the spin-angular momentum of FM. The spin accumulation dynamics observed in the nonmagnetic $\mathrm{Cu}$ layer was well explained through spin diffusion equation calculation. ${ }^{10)}$ In addition to the spin-current generation by the demagnetization inside FM layers, the spin-dependent Seebeck effect or the spin Seebeck effect at the FM/ nonmagnet interface can be a source of the spin-current. ${ }^{97-99)}$

Ballistic/diffusive spin-transport in the metallic structure enables the transfer of spin angular momentum in spin-valves where two magnetic layers are separated by a metallic nonmagnetic spacer layer as schematically shown in Fig. 4(c). This transfer of angular momentum in the spin-valve was initially observed using two ferromagnetic $[\mathrm{Co} / \mathrm{Pt}]$ layers separated by nonmagnetic $\mathrm{Ru}$ spacer layer by Malinowski et $\mathrm{al}^{20}{ }^{20}$ The speed and magnitude of the demagnetization were enhanced when two $[\mathrm{Co} / \mathrm{Pt}]$ magnetizations are antiparallel to each other, as shown in Fig. 4(d) bottom. When two magnetizations are in an anti-parallel configuration, demagnetization rates for both layers will increase because of the exchange of electrons with opposite spins between both layers, consistent with the model by Mentink et al. ${ }^{40)}$ mentioned above. In addition, no change in demagnetization dynamics was observed when two $[\mathrm{Co} / \mathrm{Pt}]$ layers were separated by insulating $\mathrm{NiO}$ as a reference sample, thereby preventing spin-transport [Fig. 4(d) top]. If the transfer of angular momentum from the other layer is more prominent than the magnetization quenching in the layer, one can expect enhancement of magnetization. Rudolf et al. observed this ultrafast magnetization enhancement in a Ni/Ru/Fe trilayer. ${ }^{24)}$ The magnetization of the bottom Fe layer [red symbols in Fig. 4(e)] increased whereas the magnetization of the top Ni layer [blue symbols in Fig. 4(e)] decreased. This enhancement in Fe magnetization was discussed based on the superdiffusive spin-transport from the $\mathrm{Ni}$ layer. In addition to the ultrafast demagnetization dynamics in spin-valves due to the angular momentum transfer, ultrafast spin-transport has been used to create ultrafast spin-transfer torques using two FM layers with noncollinear magnetization separated by a non-magnetic spacer layer. ${ }^{10,26,97,100,101)}$ This can be used 
(a)
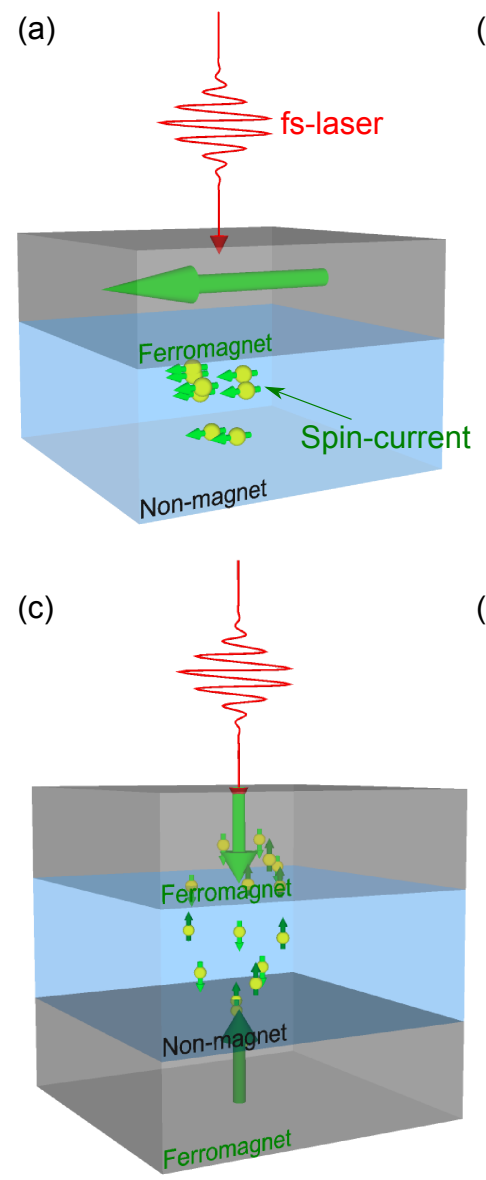

(b)

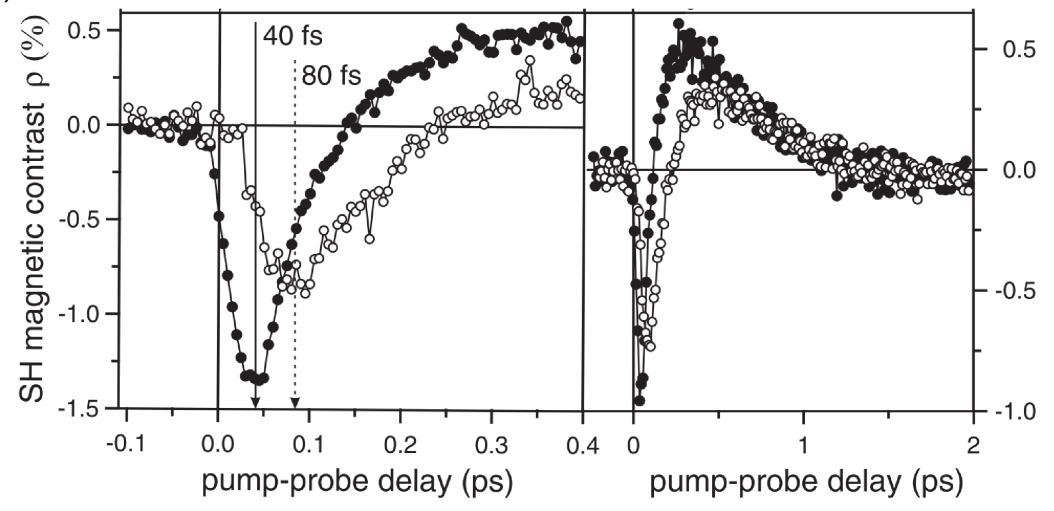

(d)

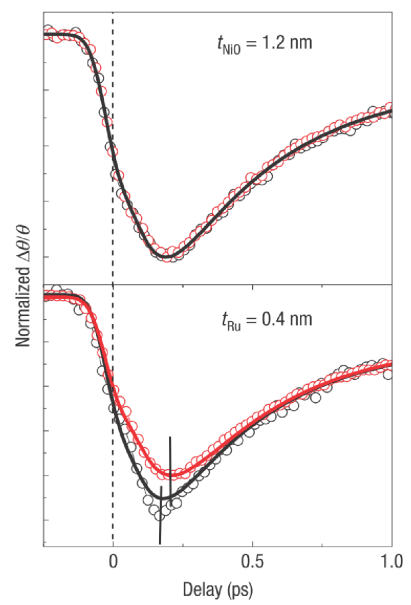

(e)

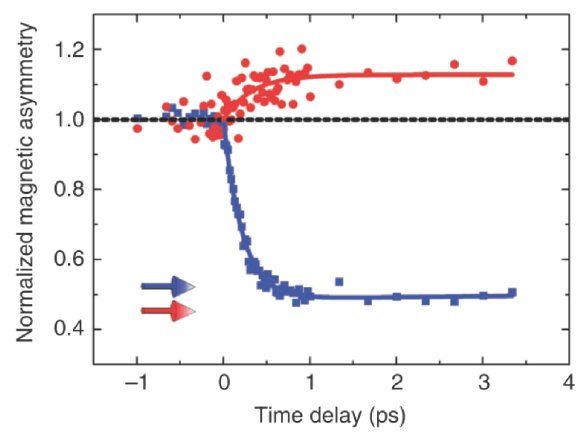

Fig. 4. (Color online) (a) Schematic illustration of fs laser induced spin-current in a FM/nonmagnet bilayer. Laser-excited hot-carrier induces spin accumulation in a non-magnetic layer. (b) Spin accumulation dynamics in non-magnetic Au in Fe/Au bilayer detected by second harmonic probe. Reprinted with permission from Melnikov et al. ${ }^{8)}$ (c) 2011 American Physical Society. (c) Schematic illustration of interlayer transfer of spin angular momentum mediated by fs-laser-induced spin-transport. (d) Increase in speed and magnitude of demagnetization in anti-parallel magnetization configuration case due to interlayer transfer of angular momentum (bottom). Comparison with reference sample that prevented spin transport (top). Reprinted with permission from Malinowski et al. ${ }^{20)} \Subset 2008$ Nature Publishing Group. (e) Enhancement in Fe magnetization in Fe/Ru/Ni trilayer. Reprinted with permission from Rudolf et al. ${ }^{24)}\left({ }^{\circ} 2012\right.$ Nature Publishing Group.

in detecting high-frequency ferromagnetic resonances and studying spin-transport.

Most previous studies focused on the ultrafast spin-current generation from a FM layer. However, spin-current generation from ferrimagnets or antiferromagnets has not been investigated intensively. Choi et al. studied spin-current generation from RE-TM ferrimagnets such as GFC and TbFeCo, ${ }^{101)}$ as schematically shown in Fig. 5(a). Ultrafast demagnetization and the spin-dependent Seebeck effect were considered as sources of spin. If we consider that the spin is generated by the change in magnetization of the FMs [Eq. (8)], then two polarities of spin exist in the generated spin-current. In this case, the directions of the spin angular momentum and magnetization are assumed to be the same for simplicity, although they must be strictly opposite. One polarity occurs when the spins are parallel to the magnetization due to the demagnetization of the FM. Another is when the spins are anti-parallel to the magnetization due to the remagnetization of the FM. Typically, the former is larger than the latter because the demagnetization speed is faster than the remagnetization speed. Figure 5(b) shows the spin accumulation in $\mathrm{Cu}$ generated using RE-TM ferrimagnets for different RE elements and compositions. The positive (negative) peak is parallel to the TM (RE) magnetic moment. It was discovered that the negative peak increased when Gd was used and the Gd concentration was increased. The slower negative contribution was explained due to the slower demagnetization of $\mathrm{Gd}$. This result implies that spin accumulation was induced by the intra-atomic exchange coupling between the conduction electron spins and localized magnetic moments in $\mathrm{Gd}$.

\section{Single-shot AOS Using Spin-valves}

The concept of single-shot AOS using a spin-valve is shown in Fig. 6(a). In this study, we focused on the GFC/ $\mathrm{Cu} / \mathrm{FM}$ structure, in which we assumed that the transfer of angular momentum in the spin-valve contributes significantly to the realization of single-shot AOS in FMs. Figure 6(b) shows the MOKE hysteresis loop observed for a GFC(5)/ $\mathrm{Cu}(9.3) /[\mathrm{Co}(0.6) / \mathrm{Pt}(1)]_{4}(\mathrm{CoPt})$ spin-valve (units of thickness: $\mathrm{nm}$ ). In this experiment, the composition of GFC used was $\mathrm{Gd}_{23.3}\left(\mathrm{Fe}_{87} \mathrm{Co}_{13}\right)_{76.7}$. The lower and higher coercive fields correspond to the magnetization reversal of the GFC and CoPt layers, respectively. A clear hysteresis for the GFC layer was observed because MOKE was sensitive to FeCo magnetic moment, ${ }^{102}$ although magnetization is mostly 
(a)

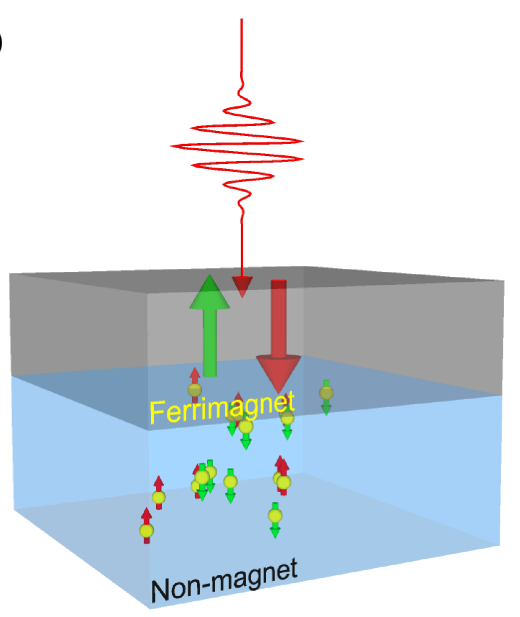

(b)

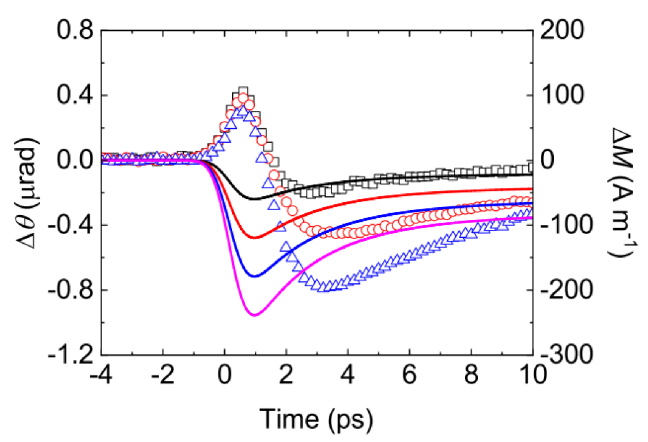

Fig. 5. (Color online) (a) Schematic illustration of ultrafast spin-current generation using ferrimagnet/nonmagnet structure. (b) Spin accumulation dynamics in non-magnetic $\mathrm{Cu}$ layer observed using RE-TM ferrimagnet/Cu bilayer. Open squares, circles, and triangles denote spin-accumulation signals generated from 20-nm-thick RE-TM ferrimagnetic $\mathrm{Tb}_{20} \mathrm{Fe}_{62} \mathrm{Co}_{18}, \mathrm{Gd}_{22} \mathrm{Fe}_{68.5}$ $\mathrm{Co}_{9.5}$, and $\mathrm{Gd}_{25} \mathrm{Fe}_{65.6} \mathrm{Co}_{9.4}$ layers, respectively. Reprinted with permission from Choi et al. ${ }^{101)}$ (C) 2018 American Physical Society.

compensated. The open red and blue symbols are the minor loops indicating the magnetization reversal of the GFC layer. No shift in the minor loop indicates that there is no coupling between the two layers owing to the thick $\mathrm{Cu}$ layer. Subsequently, we define four magnetic configurations where CoPt magnetization is pointing along the positive (+) and negative (-) directions with parallel (P) and antiparallel (AP) configurations between CoPt and FeCo in GFC. Figure 7(a) shows the MOKE images obtained after the irradiation of linearly polarized single $35 \mathrm{fs}$ laser pulses. It is noteworthy that the MOKE images were obtained from the CoPt side, whereas the fs laser pulses were irradiated from the GFC side. The magnetization configuration was changed from $\mathrm{P}+$ to $\mathrm{AP}+$, corresponding to the switching of only GFC, when a low pulse energy $E_{\mathrm{p}}$ was used [Fig. 7(a) top]. The region around the center of the spot, where the magnetization configuration was changed from $\mathrm{P}+$ to $\mathrm{P}-$, corresponds to the switching of both GFC and CoPt when $E_{\mathrm{p}}$ was increased [Fig. 7(a) middle]. When a large $E_{\mathrm{p}}$ was used, a multidomain configuration was formed around the center of the spot [Fig. 7(a) bottom] due to the large energy density. In the following, two radii are discussed, where $r_{\mathrm{GFC}}$ and $r_{\mathrm{GFC} / \mathrm{CoPt}}$ denote the radii of the switching area for the GFC layer and both the GFC and CoPt layers, respectively. Furthermore, $r_{\mathrm{GFC} / \mathrm{CoPt}}$ and $r_{\mathrm{GFC}}$ were plotted as a function of the irradiated pulse energy, as shown in Fig. 7(b). The energy dependence

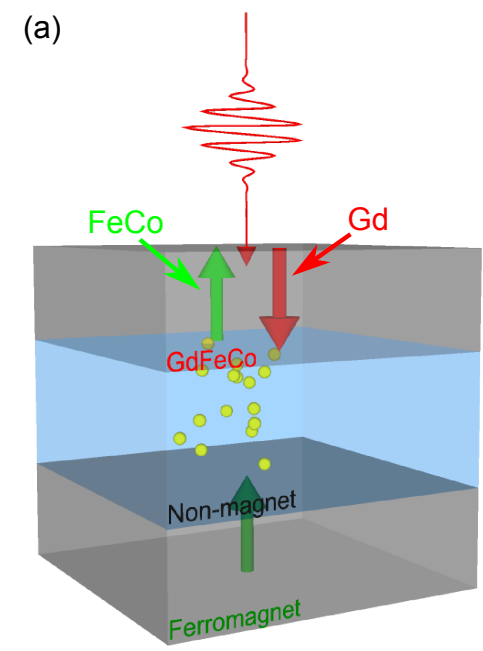

(b)

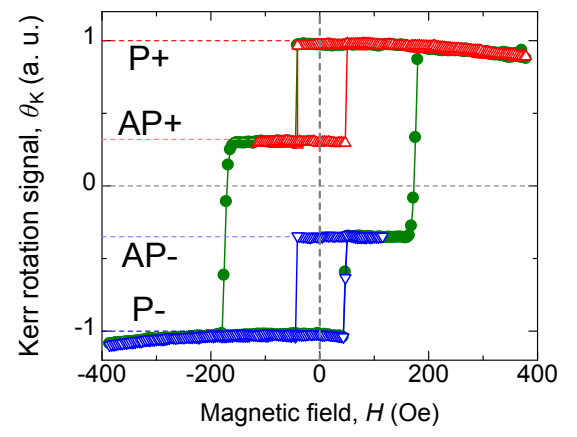

Fig. 6. (Color online) (a) Schematic illustration of concept for spintransport mediated all-optical switching in GdFeCo/non-magnet/ ferromagnet trilayer. (b) Magneto-optical Kerr effect hysteresis loop obtained for $\mathrm{FeCo}$-rich $\mathrm{GdFeCo} / \mathrm{Cu} /[\mathrm{Co} / \mathrm{Pt}]_{4}$ trilayer. Adapted from Iihama et al. ${ }^{69)}$ (C) 2018 WILEY-VCH Verlag $\mathrm{GmbH} \&$ Co. KGaA, Weinheim.

of the switching area can be explained by the Gaussian intensity distribution of the laser pulse as

$$
F_{\mathrm{th}, i}=\frac{2 E_{\mathrm{p}}}{\pi w_{0}^{2}} \exp \left(-2 r_{i}^{2} / w_{0}^{2}\right),
$$

where, $F_{\mathrm{th}, i}$ and $w_{0}$ are the threshold fluences for $r_{i}$ and the $e^{-2}$ spot size, respectively. The fitted results are shown as solid curves in Fig. 7(b). The evaluated $F_{\mathrm{th}, i}$ values for the GFC and GFC/CoPt switching were $3.2 \pm 0.7$ and $11.4 \pm$ $1.9 \mathrm{~mJ} / \mathrm{cm}^{2}$, respectively.

Interestingly, the CoPt layer was switched by the irradiation of linearly polarized single fs laser pulses. The switching of CoPt was suppressed when a spin-scattering Pt layer was inserted in the $\mathrm{Cu}$ spacer layer, ${ }^{69)}$ indicating that the CoPt switching was mediated by the spin-transport from the GFC layer. Subsequently, it was discovered that the Gd concentration significantly affected the realization of singleshot AOS in the FM layer and reduced the threshold fluences. The concept of AOS using a Gd-rich GFC is shown in Fig. 8(a). In this experiment, the composition of Gd used was $33 \%$, where a single GFC layer cannot exhibit single-shot AOS. ${ }^{70)}$ The MOKE hysteresis loop for the Gd-rich GFC/ $\mathrm{Cu} / \mathrm{CoPt}$ spin-valve is shown in Fig. 8(b). The Gd-rich GFC resulted in an inverted loop because GFC magnetization is aligned with the Gd sublattice magnetization. The MOKE 
(a)

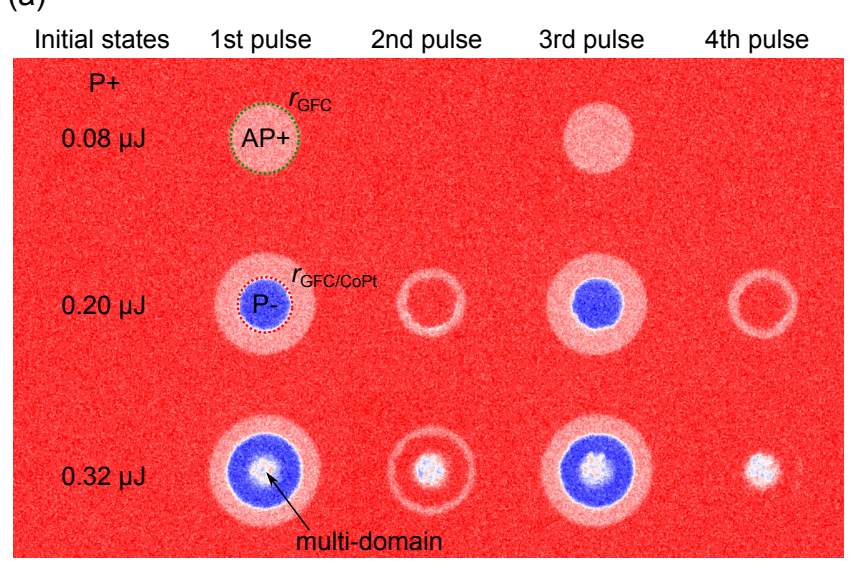

(b)

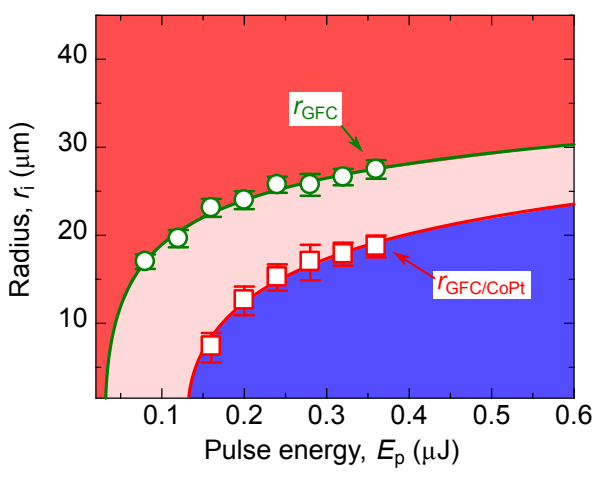

Fig. 7. (Color online) (a) MOKE images after irradiation of single fs laser pulses on $\mathrm{GdFeCo} / \mathrm{Cu} /[\mathrm{Co} / \mathrm{Pt}]$ spin-valve, where the initial configuration was $\mathrm{P}+$. (b) Radii $\left(r_{\mathrm{GFC}}, r_{\mathrm{GFC} / \mathrm{CoPt}}\right)$ evaluated by image analysis plotted as a function of the irradiated pulse energy $E_{\mathrm{p}}$. Solid curves show fitted results based on Gaussian spatial intensity distribution of the laser pulse. $e^{-2}$ spot size and threshold fluence evaluated by the fitting were $50 \pm 3(54 \pm 3) \mu \mathrm{m}$ and $3.2 \pm 0.7(11.4 \pm 1.9) \mathrm{mJ} / \mathrm{cm}^{2}$ for $r_{\mathrm{GFC}}\left(r_{\mathrm{GFC} / \mathrm{CoPt}}\right)$, respectively. Adapted from Iihama et al. ${ }^{69)}$ (C) 2018 WILEY-VCH Verlag GmbH \& Co. KGaA, Weinheim.

images after irradiation with fs laser pulses are shown in Fig. 8(c). In addition to both GFC/CoPt switching around the center of the spot, only CoPt switching was observed around the rim of the spot, unlike the feature exhibited in FeCo-rich GFC/Cu/CoPt spin-valve as shown in Fig. 7. Two different radii plotted as a function of $E_{\mathrm{p}}$ are shown in Fig. 8(d). Using the Gaussian intensity distribution analysis as mentioned above, the threshold fluence $F_{\text {th }}$ was evaluated. The $F_{\text {th }}$ for CoPt and GFC/CoPt switching were obtained as $1.65 \pm 0.19$ and $2.66 \pm 0.31 \mathrm{~mJ} / \mathrm{cm}^{2}$, respectively. It was discovered that the $F_{\text {th }}$ for CoPt switching decreases with increasing Gd content. The result above is consistent with the study of Choi et al., in which an increase in the Gd concentration increased the spin-current that is parallel to the magnetic moment of $\mathrm{Gd} .{ }^{101)}$

It was experimentally discovered that the spin-current generated from Gd in GFC was fundamental for achieving single-shot AOS in FMs. However, many questions remain regarding FM switching. For example, what are the amounts of spin-currents and heat required to switch the FM layer? Can we estimate the threshold fluence for FM switching theoretically? To answer these questions, we investigated single-shot AOS systematically by changing the $T_{\mathrm{C}}$ of the FM. ${ }^{71)}$ We chose $\left[\mathrm{Co}_{x} \mathrm{Ni}_{1-x} / \mathrm{Pt}\right]$ multilayers as a model FM material that exhibits different $T_{\mathrm{C}}$ for different $\mathrm{CoNi}$ compositions while maintaining a perpendicular easy axis. The FM switching for various compositions was observed, and it was observed that $F_{\text {th }}$ increased with the $T_{\mathrm{C}}$ of the FM layer [solid symbols in Fig. 9(a)]. One can visualize that the magnetization of FMs with low $T_{\mathrm{C}}$ can be quenched by laser pulses with less energy, thereby decreasing $F_{\text {th }}$ for the switching. To explain the threshold energy for FM switching, we developed an interface quenching criterion model. In this model, we assume that FM switching occurs when the transferred spin angular momentum from the GFC layer is greater than the magnetic moment at the interface reduced by the laser heating. First, the ultrafast demagnetization in the multilayer stack was calculated using the M3TM. The timeevolution of the electron and phonon temperatures can be described using TTM as follows: ${ }^{16)}$

$$
\begin{aligned}
C_{\mathrm{e}}\left(T_{\mathrm{e}}\right) \frac{\partial T_{\mathrm{e}}}{\partial t} & =g_{\mathrm{ep}}\left(T_{\mathrm{p}}-T_{\mathrm{e}}\right)+\frac{\partial}{\partial z}\left(\kappa_{\mathrm{e}} \frac{\partial}{\partial z} T_{\mathrm{e}}\right)+P(z, t), \\
C_{\mathrm{p}} \frac{\partial T_{\mathrm{p}}}{\partial t} & =-g_{\mathrm{ep}}\left(T_{\mathrm{p}}-T_{\mathrm{e}}\right),
\end{aligned}
$$

where, $T_{\mathrm{e}}, T_{\mathrm{p}}, C_{\mathrm{e}}, C_{\mathrm{p}}, g_{\mathrm{ep}}, \kappa_{\mathrm{e}}$, and $P(z, t)$ are the electron temperature, phonon temperature, electronic specific heat, phonon specific heat, electron-phonon coupling constant, thermal conductivity of electrons, and energy supplied by the laser pulse, respectively. $C_{\mathrm{e}}$ and $\kappa_{\mathrm{e}}$ are temperature dependent and given by, $C_{\mathrm{e}}=\gamma_{\mathrm{e}} T_{\mathrm{e}}$ and $\kappa_{\mathrm{e}}=\kappa_{0} T_{\mathrm{e}} / T_{\mathrm{p}}$, respectively, where $\gamma_{\mathrm{e}}$ is the electronic heat coefficient. $P(z, t)$ can be calculated using the light absorption profile in the metallic multilayer as follows:

$$
P(z, t)=A(z) \cdot \frac{F_{\mathrm{p}}}{\sqrt{2 \pi} \sigma} \exp \left(-\frac{t^{2}}{2 \sigma^{2}}\right),
$$

where, $A(z)$ is the light absorption profile calculated using the transfer matrix method. $F_{\mathrm{p}}$ and $\sigma$ are the fluence and pulse duration, respectively. The magnetization in the M3TM can be calculated by using the following formula: ${ }^{73)}$

$$
\frac{d m}{d t}=R m \frac{T_{\mathrm{p}}}{T_{\mathrm{C}}}\left[1-m \operatorname{coth}\left(\frac{m T_{\mathrm{C}}}{T_{\mathrm{e}}}\right)\right],
$$

where $m$ and $R$ are the normalized magnetization [= $M(t) / M_{\mathrm{s}}$, where $M_{\mathrm{s}}$ is saturation magnetization] and a constant that characterizes the demagnetization rate. $R$ is expressed as

$$
R=\frac{8 g_{\mathrm{ep}} a_{\mathrm{sf}} T_{\mathrm{C}}^{2} V_{\mathrm{at}}}{k_{\mathrm{B}} T_{\mathrm{D}}^{2} \mu / \mu_{\mathrm{B}}},
$$

where $V_{\mathrm{at}}, T_{\mathrm{D}}, k_{\mathrm{B}}$, and $\mu_{\mathrm{B}}$ are the atomic volume, the Debye temperature, the Boltzmann constant, and the Bohr magneton, respectively. The calculated magnetization of the $\mathrm{Cu} / \mathrm{FM}$ interface at $1 \mathrm{ps}$ as a function of laser fluence is shown in Fig. 9(b). The magnitude of the spin angular momentum transferred from GFC was estimated from Choi et al. ${ }^{101)}$ Choi et al. detected maximum spin accumulation in $\mathrm{Cu} \sim 100$ $\mathrm{A} / \mathrm{m}$ generated by the $\mathrm{Gd}$ sublattice using $\mathrm{Gd}_{22} \mathrm{Fe}_{68.5} \mathrm{Co}_{9.5} /$ $\mathrm{Cu}$ samples with the laser fluence of $1 \mathrm{~mJ} / \mathrm{cm}^{2}$ [red circles in Fig. 5(b)]. We used $\sim 10 \mathrm{~mJ} / \mathrm{cm}^{2}$ for the switching threshold, therefore, spin-current in the unit of magnetization $\Delta M$ generated by GFC was assumed to be proportional to the fluence and roughly calculated to be $\sim 1 \mathrm{kA} / \mathrm{m}$. Then, the interface quenching criterion is obtained using the following 
(a)

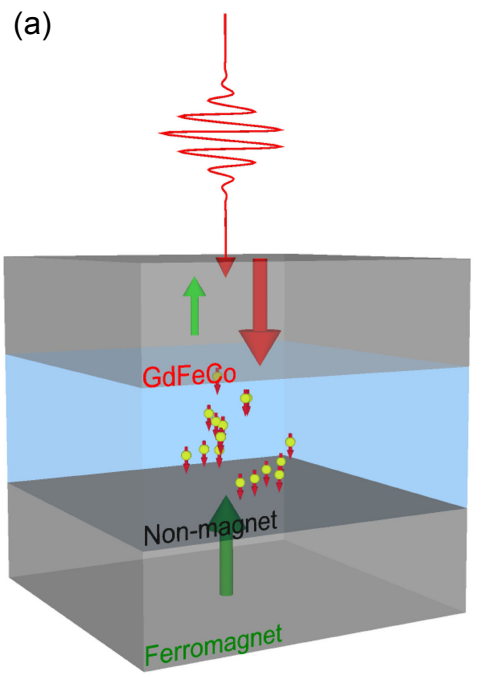

(b)

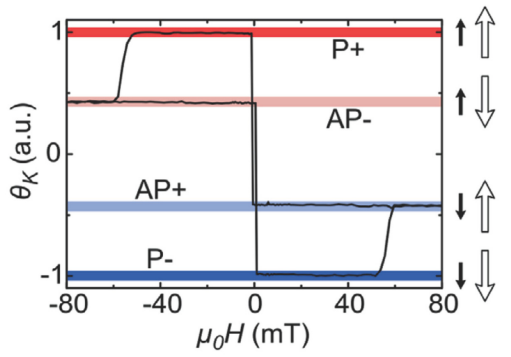

(c)
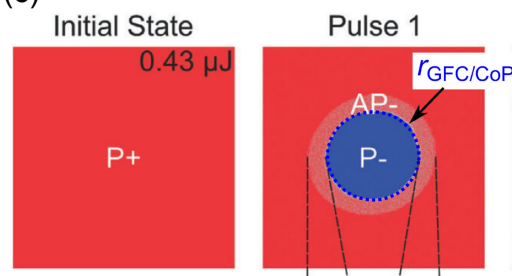

Pulse 2
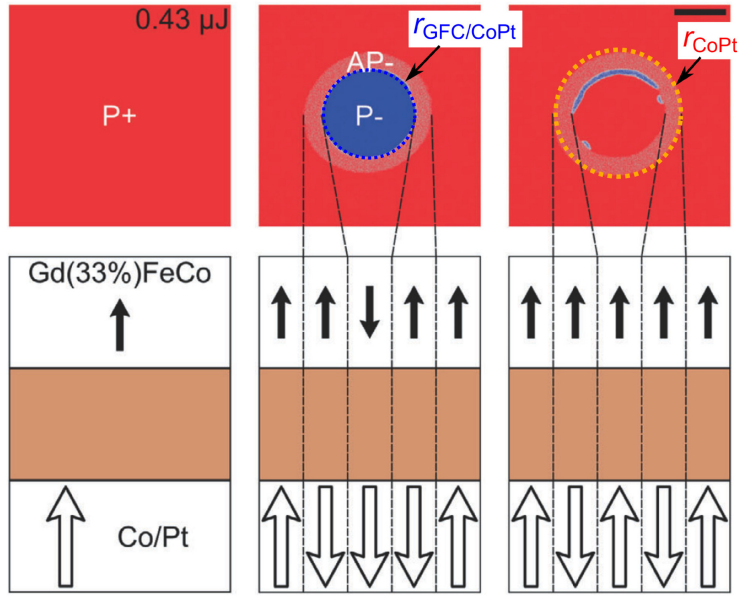

(d)

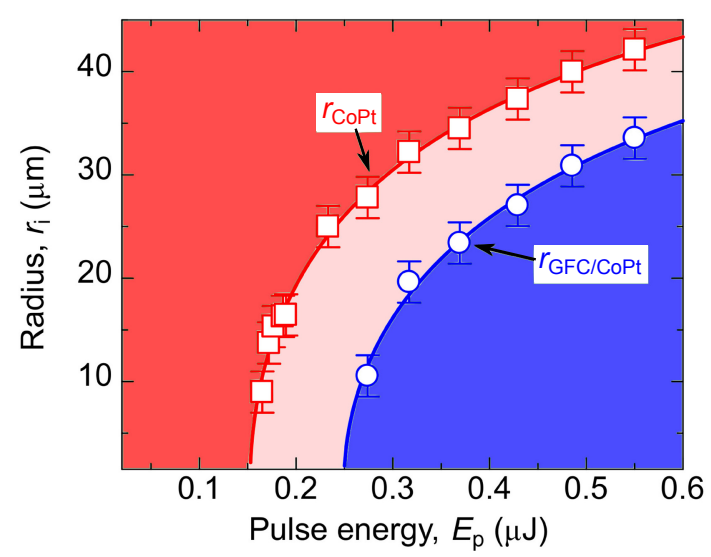

Fig. 8. (Color online) (a) Schematic illustration of spin-valve structure with Gd-rich GdFeCo. Spin-current generated by Gd sublattice transferred its angular momentum to ferromagnetic layer. (b) MOKE hysteresis loop for spin-valve with Gd-rich GdFeCo. (c) MOKE images after irradiation of fs laser pulses. (d) Radii plotted as a function of irradiated pulse energy for spin-valve with Gd-rich GdFeCo. Evaluated threshold fluence was $2.66 \pm 0.31$ (1.65 \pm 0.19 ) $\mathrm{mJ} / \mathrm{cm}^{2}$ for both GFC/CoPt (CoPt) switching. Adapted from Remy et al. ${ }^{70)}$ (C) 2020 Authors.

relation: $M_{\mathrm{s}} m_{\mathrm{c}}=\Delta M$, where $m_{\mathrm{c}}$ is the interface magnetization criterion. The interface magnetization criterion $m_{\mathrm{c}}$ is shown as horizontal lines in the inset of Fig. 9(b). The criterion differs for different FM materials because $M_{\mathrm{s}}$ for different FM materials are different. The calculated threshold fluence as a function of the $T_{\mathrm{C}}$ of the FM materials is shown as open symbols in Fig. 9(a). The trend of the experimental results is well explained by this interface quenching criterion model, although the estimation of the transferred spin-angular momentum is very rough. To explain the spin-currentmediated FM switching more precisely, improved models are required.

Recently, the theory of ultrafast magnetization dynamics in a metallic heterostructure that considers the non-local spintransport of $s$ electrons owing to the $s-d$ exchange coupling has been developed, ${ }^{103)}$ as schematically shown in Fig. 10(a). The demagnetization associated with the $s-d$ exchange coupling was initially discussed theoretically in Refs. 104 and 105. In this model, the $s$ - and $d$-electron spins are considered within the spin chemical potential $\mu_{\mathrm{s}}$ and as a localized magnetization $m_{\mathrm{d}}$. The equations of motion for $\mu_{\mathrm{s}}$ and $m_{\mathrm{d}}$ are as follows: ${ }^{103)}$

$$
\begin{aligned}
\frac{d \mu_{\mathrm{s}}}{d t} & =\rho_{\mathrm{sd}} \frac{d m_{\mathrm{d}}}{d t}-\frac{\mu_{\mathrm{s}}}{\tau_{\mathrm{s}}}, \\
\frac{d m_{\mathrm{d}}}{d t} & =\frac{1}{\tau_{\mathrm{sd}}}\left(m_{\mathrm{d}}-\frac{\mu_{\mathrm{s}}}{2 k_{\mathrm{B}} T_{\mathrm{C}}}\right)\left[1-m_{\mathrm{d}} \operatorname{coth}\left(\frac{2 m_{\mathrm{d}} k_{\mathrm{B}} T_{\mathrm{C}}-\mu_{\mathrm{s}}}{2 k_{\mathrm{B}} T_{\mathrm{e}}}\right)\right],
\end{aligned}
$$

where $\rho_{\text {sd }}$ and $\tau_{\text {sd }}^{-1}$ which are respectively expressed as $\rho_{\text {sd }}=$ $\bar{D}^{-1}-J_{\mathrm{sd}} / 2$, and $\tau_{\mathrm{sd}}^{-1}=(\pi / \hbar) J_{\mathrm{sd}}^{2} D_{\uparrow} D_{\downarrow} k_{\mathrm{B}} T_{\mathrm{C}}$, where $\bar{D}$ is 
(a)

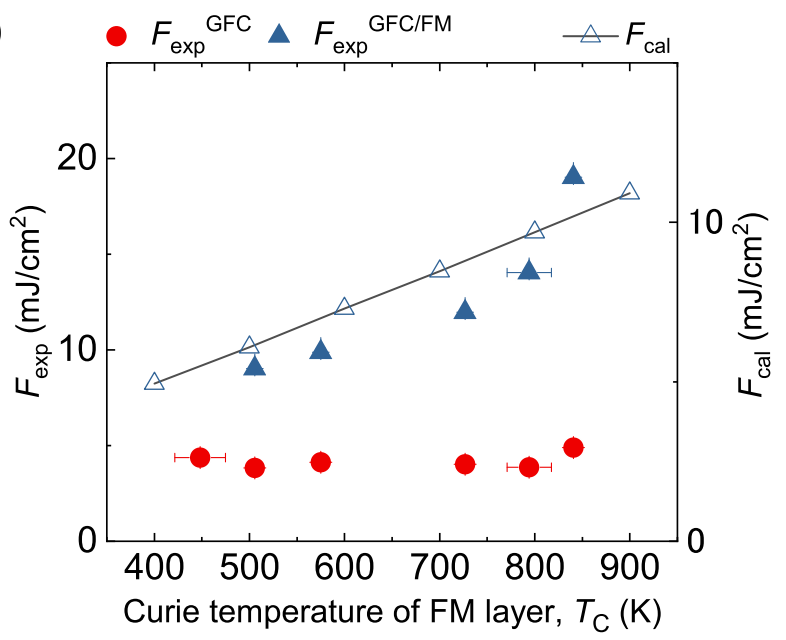

(b)

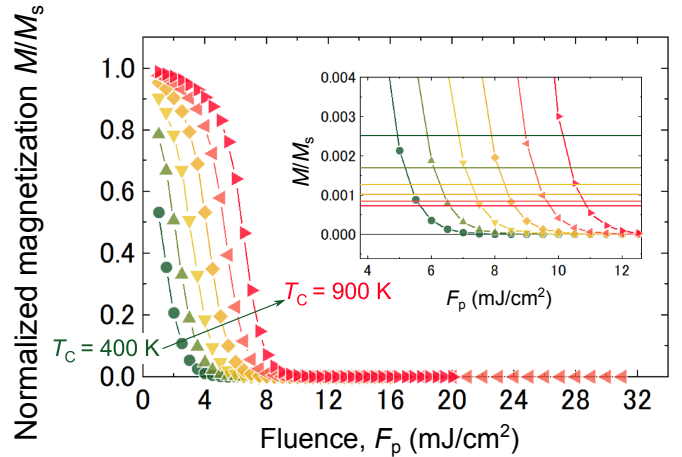

Fig. 9. (Color online) (a) Comparison of experimentally evaluated threshold fluence for FM switching and calculated fluence based on interface quenching criterion model. (b) Normalized magnetization of FM layer calculated using M3TM plotted as a function of fluence for different Curie temperatures. In interface quenching criterion model, magnetization was switched when magnetization at the $\mathrm{Cu} / \mathrm{FM}$ interface was sufficiently quenched due to laser heating. Criterion for switching is shown in the inset. Adapted from Igarashi et al. ${ }^{71)}$ (C) 2020 American Chemical Society.

defined as $\bar{D}=2 D_{\uparrow} D_{\downarrow} /\left(D_{\uparrow}+D_{\downarrow}\right)$. $D_{\uparrow(\downarrow)}$ and $J_{\text {sd }}$ are the density of states at the Fermi level for up (down) spins and the $s-d$ exchange coupling constant, respectively. $\mu_{\mathrm{s}}$ can be used to characterize the spin-transport. For example, Eq. (14) can be directly combined with the spin diffusion equation [Eq. (7)] if the diffusive transport of spin is considered. The enhancement of the speed and magnitude of demagnetization in the anti-parallel magnetization configuration in the spinvalve reported by Malinowski et al. [Fig. 4(d)] was well reproduced in this $s-d$ model calculation, as shown in Fig. 10(b). The $s-d$ exchange coupling-based calculation will be valuable for modeling single-shot AOS in the spin-valve. The GFC and CoPt can be coupled dynamically through intra-atomic exchange, such as coupling between localized $4 f$ electrons and conduction electron spin in Gd as schematically shown in Fig. 10(c). This $s-d(s-f)$ model-based calculation considering intra-atomic exchange coupling combined with TTM, light absorption profile, and spin-transport can be a model that precisely describes the single-shot AOS in a metallic spin-valve structure.

\section{Perspectives}

Numerous studies have reported that the magnetization of metallic thin films can be switched by the irradiation of a (a)

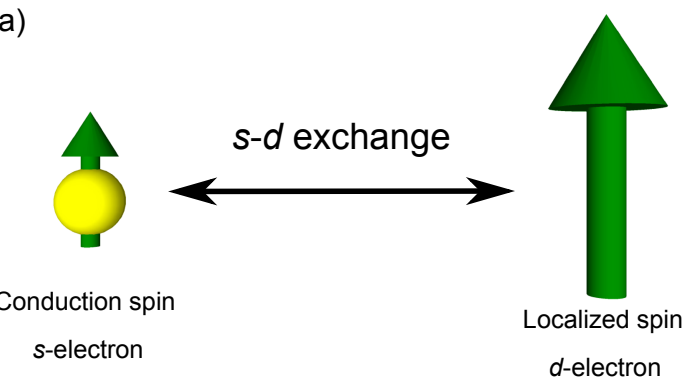

(b)

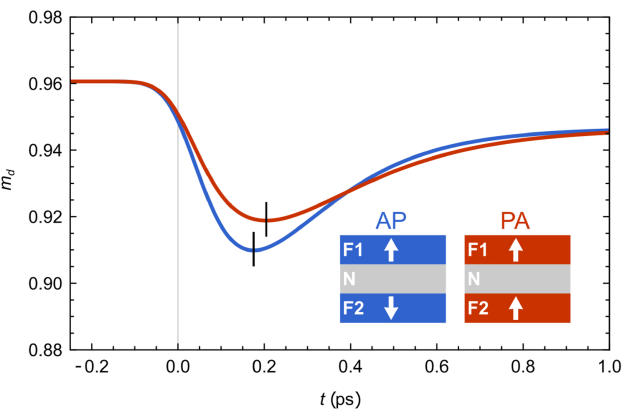

(c)

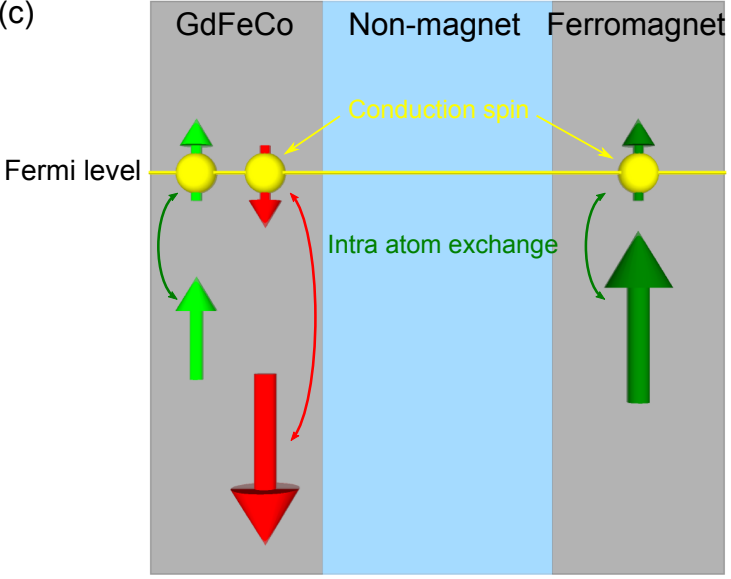

Fig. 10. (Color online) (a) Schematic illustration of $s-d$ exchange coupling. Localized magnetic moment of $d$-electrons coupled to conductive $s$ electrons that travels across metallic multilayer films. (b) Demagnetization dynamics for structures similar to those in Malinowski et al. ${ }^{20)}$ based on the $s-d$ coupling model. Reprinted with permission from Been et al. ${ }^{103)} \odot 2020$ American Physical Society. (c) Schematic illustration of dynamical exchange coupling through spin transport in spin-valve. Vertical axis indicates the electron energy and horizontal axis corresponds to the coordinate along sample thickness direction. Intra-atomic exchange coupling such as $s-d$ and $s-f$ exchange coupling enables coupling of conduction electron spin and localized magnetic moment.

single linearly polarized laser pulse without the use of an external magnetic field. Table I shows a list of single-shot AOS in metallic thin films observed using various thin-film metallic magnets and experimental techniques. It has been demonstrated that various types of magnetic materials can exhibit single-shot AOS, including FMs, although GFC was initially investigated intensively. Recently, single-shot AOS in Mn-based ferrimagnet $\mathrm{Mn}_{2} \mathrm{RuGa}$ was demonstrated. ${ }^{44)}$ The switching was attributed to the inequivalent Mn sublattices. This result indicates that single-shot AOS was not limited to RE-based ferrimagnet. The mechanism of single-shot AOS in $\mathrm{Mn}_{2} \mathrm{RuGa}$ is similar to that of GFC, although switching was realized only when the initial temperature was below the magnetization compensation temperature of $\mathrm{Mn}_{2} \mathrm{RuGa}^{45}$ ) Single-shot AOS is of purely thermal origin; therefore, 
Table I. Reports for single shot AOS of metallic thin films. Thicknesses in parentheses of the stack structure column are shown in nm.

\begin{tabular}{|c|c|c|c|}
\hline Materials & Stack structure & Experiment/Remarks & Reference \\
\hline \multirow[t]{13}{*}{ GdFeCo Ferri. } & $\mathrm{SiN}(100) / \mathrm{GdFeCo}(30) / \mathrm{SiN}(60)$ & $\begin{array}{l}\text { Time- and element-resolved experiment using X-ray } \\
\text { magnetic circular dichroism (XMCD) }\end{array}$ & Radu et al. ${ }^{30)}$ \\
\hline & $\mathrm{SiN}(5) / \mathrm{GdFeCo}(20) / \mathrm{SiN}(3)$ & $\begin{array}{l}\text { Switching of both out-of-plane and in-plane } \\
\text { anisotropy GdFeCo films detected by XMCD }\end{array}$ & Ostler et al. ${ }^{31)}$ \\
\hline & Glass sub./AlTi(10)/SiN(5)/GdFeCo(20)/SiN(60) & Time-resolved experiment & Vahaplar et al. ${ }^{34)}$ \\
\hline & Glass sub./AlTi(10)/SiN(5)/GdFeCo(20)/SiN(60) & $\begin{array}{l}\text { Laser parameters (pulse duration, chirp, bandwidth) } \\
\text { dependence }\end{array}$ & Steil et al. ${ }^{109)}$ \\
\hline & $\operatorname{SiN}(100) / \operatorname{GdFeCo}(30) / \operatorname{SiN}(60)$ & $\begin{array}{l}\text { Time-, element-, and space-resolved experiment with } \\
\text { nanoscale resolution }\end{array}$ & Graves et al. ${ }^{37)}$ \\
\hline & Glass sub./AlTi/SiN/GdFeCo/SiN(60) & Thickness dependence & Yoshikawa et al. ${ }^{110)}$ \\
\hline & Glass sub./AlTi(10)/SiN(5)/GdFeCo(20)/SiN(60) & Composition dependence & Yoshikawa et al. ${ }^{111)}$ \\
\hline & $\operatorname{GdFeCo}(20)$ & Detection using anomalous Hall effect & Hadri et al. ${ }^{55)}$ \\
\hline & $\mathrm{MgO} / \mathrm{GdFeCo}(20) / \mathrm{Ta}(5)$ & Detection using tunnel magnetoresistance & Chen et al. ${ }^{112)}$ \\
\hline & $\mathrm{Ta}(2.5) / \mathrm{GdFeCo}(14) / \mathrm{Ta}(3.6) / \mathrm{Ta}_{2} \mathrm{O}_{5}(2.8)$ & Pulse duration and temperature dependence & Gorchon et al. ${ }^{35)}$ \\
\hline & $\mathrm{GdFeCo}(6) / \mathrm{Au}(113) / \mathrm{Pt}(6)$ & Switching induced by electronic heat current pulse & Wilson et al. ${ }^{95)}$ \\
\hline & $\mathrm{GdFeCo}(20)$ & $\begin{array}{l}\text { Switching induced by electrical pulses generated by } \\
\text { photoconductive switches }\end{array}$ & Yang et al. ${ }^{106)}$ \\
\hline & $\mathrm{GdFeCo}(10) / \mathrm{SiN}(80) / \mathrm{GdFeCo}(10)$ & Selective switching by rotating polarization axis & Ignatyeva et al. ${ }^{107)}$ \\
\hline GdCo Ferri. & $\mathrm{Ta}(3) / \mathrm{Pt}(3) / \mathrm{GdCo}(10) / \mathrm{Pt}(3)$ & Switching of micropatterned dot arrays & Ghazaly et al. ${ }^{113)}$ \\
\hline TbFeCo Ferri. & $\mathrm{SiN} / \mathrm{TbFeCo} / \mathrm{SiN}$ & $\begin{array}{l}\text { Local optical field enhancement due to plasmonic } \\
\text { effect }\end{array}$ & Liu et al. ${ }^{42)}$ \\
\hline Gd/Co synthetic Ferri. & $\mathrm{Ta}(4) / \mathrm{Pt}(4) / \mathrm{Co} / \mathrm{Gd}(3) / \mathrm{Pt}(2)$ & Co thickness dependence & Lalieu et al. ${ }^{46)}$ \\
\hline $\mathrm{Tb} /$ Co synthetic Ferri. & $\mathrm{CoFeB} / \mathrm{Ta} /[\mathrm{Tb} / \mathrm{Co}]_{5}$ & $\begin{array}{l}\text { Switching of structure for magnetic tunnel junction } \\
\text { device }\end{array}$ & Aviles-Felix et al. $^{43)}$ \\
\hline \multirow[t]{2}{*}{$\mathrm{Mn}_{2} \mathrm{RuGa}$ Ferri. } & $\mathrm{Mn}_{2} \mathrm{RuGa}$ film on $\mathrm{MgO}$ single crystal sub. & Inequivalent sublattices of two $\mathrm{Mn}$ atoms & Banerjee et al. ${ }^{44)}$ \\
\hline & $\mathrm{Mn}_{2} \mathrm{Ru}_{0.8} \mathrm{Ga}(32), \mathrm{Mn}_{2} \mathrm{Ru}_{0.75} \mathrm{Ga}(18)$ on $\mathrm{MgO}$ sub. & Temperature and pulse duration dependence & Davies et al. $^{45)}$ \\
\hline Co/Pt Ferro. & $\begin{array}{l}\mathrm{Ta}(5) / \mathrm{GdFeCo}(20) / \mathrm{Co}(0.4) / \mathrm{Pt} / \mathrm{Co}(0.6) / \mathrm{Pt}(0.7) / \\
\mathrm{Co}(0.6) / \operatorname{Pt}(3)\end{array}$ & CoPt switching due to coupling with GdFeCo & Gorchon et al. ${ }^{68)}$ \\
\hline $\mathrm{Pt} / \mathrm{Co} / \mathrm{Pt}$ Ferro. & $\mathrm{Pt}(1.5) / \mathrm{Co}(0.7) / \mathrm{Pt}(1.5)$ & Switching due to stabilization of magnetic domains & Vomir et al. ${ }^{67)}$ \\
\hline Co/Pt Ferro. & $\mathrm{GdFeCo}(5) / \mathrm{Cu} /[\mathrm{Co}(0.6) / \mathrm{Pt}(1)]_{4}$ & Spin current mediated switching & Iihama et al. ${ }^{69)}$ \\
\hline
\end{tabular}

magnetization switching can be induced by ultrashort electrical pulses and has garnered significant attention for future ultrafast spintronics devices. Magnetization switching triggered by electrical pulses has been reported in several studies. It was demonstrated that the magnetization switching of GFC can be induced by hot electron pulses generated by the irradiation of optical pulses, propagating over a distance beyond the penetration depth of the light. ${ }^{12,95)}$ In addition, the magnetization switching of GFC with ultrashort electrical pulses generated by photoconductive switches was demonstrated. ${ }^{106)}$

Although many reports regarding single-shot AOS pertain to ferrimagnets, single-shot AOS even in FMs can be realized by using the spin-valve structure described herein. The use of ultrafast spin-transport in the spin-valve can potentially achieve single-shot AOS in various types of magnetic materials, including FMs. Moreover, the use of multiple magnetic layers without coupling between the layers enables multi-level magnetization switching, which will be promising for future three-dimensional magnetic recording. Recently, plasmonic layer-selective single-shot AOS was demonstrated using two GFC layers separated by a transparent layer. ${ }^{107)}$ The two GFCs can be switched selectively by rotating the polarization axis of linearly polarized laser pulses owing to plasmonic effects in the heterostructure.

To increase the density of the recording area and to reduce the energy required for the magnetization switching, local enhancement of optical fields arising from plasmonic effects $^{42,108)}$ must be designed for future all-optical magnetic recording.

\section{Conclusion}

The use of ultrashort laser pulses enables the study of ultrafast electron/spin dynamics in metals. The highly nonequilibrium electron/spin states driven by single fs optical pulses resulted in magnetization switching in metallic thin films, termed as single-shot AOS. Single-shot AOS was primarily investigated intensively in the GFC ferrimagnet. Recently, single-shot AOS in FMs was demonstrated using spin-transport in spin-valves. The physical mechanism of single-shot AOS in GFC, ultrafast spin-transport in a metallic structure, and recent single-shot AOS of FMs were described herein. The ultrafast optical manipulation of spin-transport and switching of nanomagnets in metallic heterostructures have potential to realize future ultrafast and energy-efficient photo-spintronics storage/memory devices.

Acknowledgment This article is based upon work from COST Action CA17123 MAGNETOFON, supported by COST (European Cooperation in Science and Technology). This work was supported by the French PIA project "Lorraine Université d'Excellence" reference ANR-15-IDEX-04-LUE, the ANR UFO ANR-20-CE09-0013. the Institut Carnot ICEEL, the Sakura Program, the JSPS Bilateral Program, JSPS KAKENHI, JP19J12926, and the Tohoku University-Université de Lorraine Matching Funds. This work is part of the project PLUS supported by the "FEDER-FSE Lorraine et Massif Vosges 2014-2020", a European Union Program and the Region Grand est. J.I. acknowledges financial support from GP-Spin of Tohoku University and JSTOPERA. 
*satoshi.iihama.d6@tohoku.ac.jp

1) E. Beaurepaire, J. C. Merle, A. Daunois, and J. Y. Bigot, Phys. Rev. Lett. 76, 4250 (1996).

2) C. Stamm, T. Kachel, N. Pontius, R. Mitzner, T. Quast, K. Holldack, S. Khan, C. Lupulescu, E. F. Aziz, M. Wietstruk, H. A. Dürr, and W. Eberhardt, Nat. Mater. 6, 740 (2007).

3) C. Boeglin, E. Beaurepaire, V. Halté, V. López-Flores, C. Stamm, N. Pontius, H. A. Dürr, and J. Y. Bigot, Nature 465, 458 (2010).

4) F. Siegrist, J. A. Gessner, M. Ossiander, C. Denker, Y. P. Chang, M. C. Schröder, A. Guggenmos, Y. Cui, J. Walowski, U. Martens, J. K. Dewhurst, U. Kleineberg, M. Münzenberg, S. Sharma, and M. Schultze, Nature 571, 240 (2019).

5) A. Kirilyuk, A. V. Kimel, and T. Rasing, Rev. Mod. Phys. 82, 2731 (2010).

6) J. Walowski and M. Münzenberg, J. Appl. Phys. 120, 140901 (2016).

7) G. Malinowski, N. Bergeard, M. Hehn, and S. Mangin, Eur. Phys. J. B 91, 98 (2018)

8) A. Melnikov, I. Razdolski, T. O. Wehling, E. T. Papaioannou, V. Roddatis, P. Fumagalli, O. Aktsipetrov, A. I. Lichtenstein, and U. Bovensiepen, Phys. Rev. Lett. 107, 076601 (2011).

9) A. Eschenlohr, M. Battiato, P. Maldonado, N. Pontius, T. Kachel, K. Holldack, R. Mitzner, A. Föhlisch, P. M. Oppeneer, and C. Stamm, Nat. Mater. 12, 332 (2013).

10) G. M. Choi, B. C. Min, K. J. Lee, and D. G. Cahill, Nat. Commun. 5, 4334 (2014).

11) N. Bergeard, M. Hehn, S. Mangin, G. Lengaigne, F. Montaigne, M. L. M. Lalieu, B. Koopmans, and G. Malinowski, Phys. Rev. Lett. 117, 147203 (2016).

12) Y. Xu, M. Deb, G. Malinowski, M. Hehn, W. Zhao, and S. Mangin, Adv. Mater. 29, 1703474 (2017).

13) T. Kampfrath, M. Battiato, P. Maldonado, G. Eilers, J. Nötzold, S. Mährlein, V. Zbarsky, F. Freimuth, Y. Mokrousov, S. Blügel, M. Wolf, I. Radu, P. M. Oppeneer, and M. Münzenberg, Nat. Nanotechnol. 8, 256 (2013).

14) T. Seifert, S. Jaiswal, U. Martens, J. Hannegan, L. Braun, P. Maldonado, F. Freimuth, A. Kronenberg, J. Henrizi, I. Radu, E. Beaurepaire, Y. Mokrousov, P. M. Oppeneer, M. Jourdan, G. Jakob, D. Turchinovich, L. M. Hayden, M. Wolf, M. Münzenberg, M. Kläui, and T. Kampfrath, Nat. Photonics 10, 483 (2016).

15) S. D. Brorson, J. G. Fujimoto, and E. P. Ippen, Phys. Rev. Lett. 59, 1962 (1987).

16) J. Hohlfeld, S. S. Wellershoff, J. Güdde, U. Conrad, V. Jähnke, and E. Matthias, Chem. Phys. 251, 237 (2000).

17) M. Bonn, D. N. Denzler, S. Funk, M. Wolf, S. S. Wellershoff, and J. Hohlfeld, Phys. Rev. B 61, 1101 (2000).

18) M. Battiato, K. Carva, and P. M. Oppeneer, Phys. Rev. Lett. 105, 027203 (2010).

19) M. Battiato, K. Carva, and P. M. Oppeneer, Phys. Rev. B 86, 024404 (2012).

20) G. Malinowski, F. Dalla Longa, J. H. H. Rietjens, P. V. Paluskar, R. Huijink, H. J. M. Swagten, and B. Koopmans, Nat. Phys. 4, 855 (2008).

21) S. Mathias, C. La-O-Vorakiat, P. Grychtol, P. Granitzka, E. Turgut, J. M. Shaw, R. Adam, H. T. Nembach, M. E. Siemens, S. Eich, C. M. Schneider, T. J. Silva, M. Aeschlimann, M. M. Murnane, and H. C. Kapteyn, Proc. Natl. Acad. Sci. U.S.A. 109, 4792 (2012).

22) M. Hofherr, S. Häuser, J. K. Dewhurst, P. Tengdin, S. Sakshath, H. T. Nembach, S. T. Weber, J. M. Shaw, T. J. Silva, H. C. Kapteyn, M. Cinchetti, B. Rethfeld, M. M. Murnane, D. Steil, B. Stadtmüller, S. Sharma, M. Aeschlimann, and S. Mathias, Sci. Adv. 6, eaay8717 (2020).

23) P. Tengdin, C. Gentry, A. Blonsky, D. Zusin, M. Gerrity, L. Hellbrück, M. Hofherr, J. Shaw, Y. Kvashnin, E. K. Delczeg-Czirjak, M. Arora, H. Nembach, T. J. Silva, S. Mathias, M. Aeschlimann, H. C. Kapteyn, D. Thonig, K. Koumpouras, O. Eriksson, and M. M. Murnane, Sci. Adv. 6, eaaz1100 (2020).

24) D. Rudolf, C. La-O-Vorakiat, M. Battiato, R. Adam, J. M. Shaw, E. Turgut, P. Maldonado, S. Mathias, P. Grychtol, H. T. Nembach, T. J. Silva, M. Aeschlimann, H. C. Kapteyn, M. M. Murnane, C. M. Schneider, and P. M. Oppeneer, Nat. Commun. 3, 1037 (2012).

25) E. Turgut, C. La-O-Vorakiat, J. M. Shaw, P. Grychtol, H. T. Nembach, D. Rudolf, R. Adam, M. Aeschlimann, C. M. Schneider, T. J. Silva, M. M. Murnane, H. C. Kapteyn, and S. Mathias, Phys.
Rev. Lett. 110, 197201 (2013).

26) A. J. Schellekens, K. C. Kuiper, R. R. J. C. De Wit, and B. Koopmans, Nat. Commun. 5, 4333 (2014).

27) A. Alekhin, I. Razdolski, N. Ilin, J. P. Meyburg, D. Diesing, V. Roddatis, I. Rungger, M. Stamenova, S. Sanvito, U. Bovensiepen, and A. Melnikov, Phys. Rev. Lett. 119, 017202 (2017).

28) A. V. Kimel and M. Li, Nat. Rev. Mater. 4, 189 (2019).

29) C. D. Stanciu, F. Hansteen, A. V. Kimel, A. Kirilyuk, A. Tsukamoto, A. Itoh, and T. Rasing, Phys. Rev. Lett. 99, 047601 (2007).

30) I. Radu, K. Vahaplar, C. Stamm, T. Kachel, N. Pontius, H. A. Dürr, T. A. Ostler, J. Barker, R. F. L. Evans, R. W. Chantrell, A. Tsukamoto, A. Itoh, A. Kirilyuk, T. Rasing, and A. V. Kimel, Nature 472, 205 (2011).

31) T. A. Ostler, J. Barker, R. F. L. Evans, R. W. Chantrell, U. Atxitia, O. Chubykalo-Fesenko, S. El Moussaoui, L. Le Guyader, E. Mengotti, L. J. Heyderman, F. Nolting, A. Tsukamoto, A. Itoh, D. Afanasiev, B. A. Ivanov, A. M. Kalashnikova, K. Vahaplar, J. Mentink, A. Kirilyuk, T. Rasing, and A. V. Kimel, Nat. Commun. 3, 666 (2012).

32) C. D. Stanciu, A. Tsukamoto, A. V. Kimel, F. Hansteen, A. Kirilyuk, A. Itoh, and T. Rasing, Phys. Rev. Lett. 99, 217204 (2007).

33) K. Vahaplar, A. M. Kalashnikova, A. V. Kimel, D. Hinzke, U. Nowak, R. Chantrell, A. Tsukamoto, A. Itoh, A. Kirilyuk, and T. Rasing, Phys. Rev. Lett. 103, 117201 (2009).

34) K. Vahaplar, A. M. Kalashnikova, A. V. Kimel, S. Gerlach, D. Hinzke, U. Nowak, R. Chantrell, A. Tsukamoto, A. Itoh, A. Kirilyuk, and T. Rasing, Phys. Rev. B 85, 104402 (2012).

35) J. Gorchon, R. B. Wilson, Y. Yang, A. Pattabi, J. Y. Chen, L. He, J. P. Wang, M. Li, and J. Bokor, Phys. Rev. B 94, 184406 (2016).

36) N. Bergeard, V. López-Flores, V. Halté, M. Hehn, C. Stamm, N. Pontius, E. Beaurepaire, and C. Boeglin, Nat. Commun. 5, 3466 (2014).

37) C. E. Graves, A. H. Reid, T. Wang, B. Wu, S. De Jong, K. Vahaplar, I. Radu, D. P. Bernstein, M. Messerschmidt, L. Müller, R. Coffee, M. Bionta, S. W. Epp, R. Hartmann, N. Kimmel, G. Hauser, A. Hartmann, P. Holl, H. Gorke, J. H. Mentink, A. Tsukamoto, A. Fognini, J. J. Turner, W. F. Schlotter, D. Rolles, H. Soltau, L. Strüder, Y. Acremann, A. V. Kimel, A. Kirilyuk, T. Rasing, J. Stöhr, A. O. Scherz, and H. A. Dürr, Nat. Mater. 12, 293 (2013).

38) L. Le Guyader, M. Savoini, S. El Moussaoui, M. Buzzi, A. Tsukamoto, A. Itoh, A. Kirilyuk, T. Rasing, A. V. Kimel, and F. Nolting, Nat. Commun. 6, 5839 (2015).

39) E. Iacocca, T. M. Liu, A. H. Reid, Z. Fu, S. Ruta, P. W. Granitzka, E. Jal, S. Bonetti, A. X. Gray, C. E. Graves, R. Kukreja, Z. Chen, D. J. Higley, T. Chase, L. Le Guyader, K. Hirsch, H. Ohldag, W. F. Schlotter, G. L. Dakovski, G. Coslovich, M. C. Hoffmann, S. Carron, A. Tsukamoto, A. Kirilyuk, A. V. Kimel, T. Rasing, J. Stöhr, R. F. L. Evans, T. Ostler, R. W. Chantrell, M. A. Hoefer, T. J. Silva, and H. A. Dürr, Nat. Commun. 10, 1756 (2019).

40) J. H. Mentink, J. Hellsvik, D. V. Afanasiev, B. A. Ivanov, A. Kirilyuk, A. V. Kimel, O. Eriksson, M. I. Katsnelson, and T. Rasing, Phys. Rev. Lett. 108, 057202 (2012).

41) S. Wienholdt, D. Hinzke, K. Carva, P. M. Oppeneer, and U. Nowak, Phys. Rev. B 88, 020406(R) (2013).

42) T. M. Liu, T. Wang, A. H. Reid, M. Savoini, X. Wu, B. Koene, P. Granitzka, C. E. Graves, D. J. Higley, Z. Chen, G. Razinskas, M. Hantschmann, A. Scherz, J. Stöhr, A. Tsukamoto, B. Hecht, A. V. Kimel, A. Kirilyuk, T. Rasing, and H. A. Dürr, Nano Lett. 15, 6862 (2015).

43) L. Avilés-Félix, A. Olivier, G. Li, C. S. Davies, L. Álvaro-Gómez, M. Rubio-Roy, S. Auffret, A. Kirilyuk, A. V. Kimel, T. Rasing, L. D. Buda-Prejbeanu, R. C. Sousa, B. Dieny, and I. L. Prejbeanu, Sci. Rep. 10, 5211 (2020).

44) C. Banerjee, N. Teichert, K. E. Siewierska, Z. Gercsi, G. Y. P. Atcheson, P. Stamenov, K. Rode, J. M. D. Coey, and J. Besbas, Nat. Commun. 11, 4444 (2020).

$45)$ C. S. Davies, G. Bonfiglio, K. Rode, J. Besbas, C. Banerjee, P. Stamenov, J. M. D. Coey, A. V. Kimel, and A. Kirilyuk, Phys. Rev. Res. 2, 032044(R) (2020).

46) M. L. M. Lalieu, M. J. G. Peeters, S. R. R. Haenen, R. Lavrijsen, and B. Koopmans, Phys. Rev. B 96, 220411(R) (2017).

47) M. L. M. Lalieu, R. Lavrijsen, and B. Koopmans, Nat. Commun. 10, 110 (2019)

48) Y. L. W. van Hees, P. van de Meugheuvel, B. Koopmans, and R. 
Lavrijsen, Nat. Commun. 11, 3835 (2020).

49) C. H. Lambert, S. Mangin, B. S. D. C. S. Varaprasad, Y. K. Takahashi, M. Hehn, M. Cinchetti, G. Malinowski, K. Hono, Y. Fainman, M. Aeschlimann, and E. E. Fullerton, Science 345, 1337 (2014).

50) Y. K. Takahashi, R. Medapalli, S. Kasai, J. Wang, K. Ishioka, S. H. Wee, O. Hellwig, K. Hono, and E. E. Fullerton, Phys. Rev. Appl. 6, 054004 (2016).

51) R. John, M. Berritta, D. Hinzke, C. Müller, T. Santos, H. Ulrichs, P. Nieves, J. Walowski, R. Mondal, O. Chubykalo-Fesenko, J. McCord, P. M. Oppeneer, U. Nowak, and M. Münzenberg, Sci. Rep. 7, 4114 (2017).

52) F. Cheng, Z. Du, X. Wang, Z. Cai, L. Li, C. Wang, A. Benabbas, P. Champion, N. Sun, L. Pan, and Y. Liu, Adv. Opt. Mater. 8, 2000379 (2020).

53) S. Alebrand, M. Gottwald, M. Hehn, D. Steil, M. Cinchetti, D. Lacour, E. E. Fullerton, M. Aeschlimann, and S. Mangin, Appl. Phys. Lett. 101, 162408 (2012)

54) S. Mangin, M. Gottwald, C. H. Lambert, D. Steil, V. Uhliř, L. Pang, M. Hehn, S. Alebrand, M. Cinchetti, G. Malinowski, Y. Fainman, M. Aeschlimann, and E. E. Fullerton, Nat. Mater. 13, 286 (2014).

55) M. S. El Hadri, P. Pirro, C. H. Lambert, S. Petit-Watelot, Y. Quessab, M. Hehn, F. Montaigne, G. Malinowski, and S. Mangin, Phys. Rev. B 94, 064412 (2016).

56) M. S. El Hadri, M. Hehn, P. Pirro, C. H. Lambert, G. Malinowski, E. E. Fullerton, and S. Mangin, Phys. Rev. B 94, 064419 (2016).

57) R. Medapalli, D. Afanasiev, D. K. Kim, Y. Quessab, S. Manna, S. A Montoya, A. Kirilyuk, T. Rasing, A. V. Kimel, and E. E. Fullerton, Phys. Rev. B 96, 224421 (2017).

58) Y. Xu, M. Hehn, W. Zhao, X. Lin, G. Malinowski, and S. Mangin, Phys. Rev. B 100, 064424 (2019).

59) A. Ciuciulkaite, K. Mishra, M. V. Moro, I. A. Chioar, R. M. RowanRobinson, S. Parchenko, A. Kleibert, B. Lindgren, G. Andersson, C. S. Davies, A. Kimel, M. Berritta, P. M. Oppeneer, A. Kirilyuk, and V. Kapaklis, Phys. Rev. Mater. 4, 104418 (2020).

60) G. Kichin, M. Hehn, J. Gorchon, G. Malinowski, J. Hohlfeld, and S Mangin, Phys. Rev. Appl. 12, 024019 (2019).

61) J. Gorchon, Y. Yang, and J. Bokor, Phys. Rev. B 94, 020409(R) (2016).

62) M. O. A. Ellis, E. E. Fullerton, and R. W. Chantrell, Sci. Rep. 6, 30522 (2016).

63) M. Berritta, R. Mondal, K. Carva, and P. M. Oppeneer, Phys. Rev. Lett. 117, 137203 (2016)

64) P. Scheid, G. Malinowski, S. Mangin, and S. Lebègue, Phys. Rev. B 100, 214402 (2019).

65) Y. Quessab, R. Medapalli, M. S. El Hadri, M. Hehn, G. Malinowski, E. E. Fullerton, and S. Mangin, Phys. Rev. B 97, 054419 (2018).

66) Y. Quessab, M. Deb, J. Gorchon, M. Hehn, G. Malinowski, and S. Mangin, Phys. Rev. B 100, 024425 (2019).

67) M. Vomir, M. Albrecht, and J. Y. Bigot, Appl. Phys. Lett. 111 242404 (2017).

68) J. Gorchon, C. H. Lambert, Y. Yang, A. Pattabi, R. B. Wilson, S. Salahuddin, and J. Bokor, Appl. Phys. Lett. 111, 042401 (2017).

69) S. Iihama, Y. Xu, M. Deb, G. Malinowski, M. Hehn, J. Gorchon, E. E. Fullerton, and S. Mangin, Adv. Mater. 30, 1804004 (2018).

70) Q. Remy, J. Igarashi, S. Iihama, G. Malinowski, M. Hehn, J. Gorchon, J. Hohlfeld, S. Fukami, H. Ohno, and S. Mangin, Adv. Sci. 7, 2001996 (2020).

71) J. Igarashi, Q. Remy, S. Iihama, G. Malinowski, M. Hehn, J. Gorchon, J. Hohlfeld, S. Fukami, H. Ohno, and S. Mangin, Nano Lett. 20, 8654 (2020).

72) A. R. Khorsand, M. Savoini, A. Kirilyuk, A. V. Kimel, A. Tsukamoto, A. Itoh, and T. Rasing, Phys. Rev. Lett. 108, 127205 (2012).

73) B. Koopmans, G. Malinowski, F. Dalla Longa, D. Steiauf, M. Fähnle, T. Roth, M. Cinchetti, and M. Aeschlimann, Nat. Mater. 9, 259 (2010).

74) J. Barker, U. Atxitia, T. A. Ostler, O. Hovorka, O. ChubykaloFesenko, and R. W. Chantrell, Sci. Rep. 3, 3262 (2013).

75) R. F. L. Evans, T. A. Ostler, R. W. Chantrell, I. Radu, and T. Rasing, Appl. Phys. Lett. 104, 082410 (2014).

76) C. Xu, T. A. Ostler, and R. W. Chantrell, Phys. Rev. B 93, 054302 (2016).
77) R. Moreno, S. Khmelevskyi, and O. Chubykalo-Fesenko, Phys. Rev. B 99, 184401 (2019)

78) U. Atxitia, D. Hinzke, and U. Nowak, J. Phys. D 50, 033003 (2017).

79) U. Atxitia, T. Ostler, J. Barker, R. F. L. Evans, R. W. Chantrell, and O. Chubykalo-Fesenko, Phys. Rev. B 87, 224417 (2013).

80) A. J. Schellekens and B. Koopmans, Phys. Rev. B 87, 020407(R) (2013).

81) M. Beens, M. L. M. Lalieu, A. J. M. Deenen, R. A. Duine, and B. Koopmans, Phys. Rev. B 100, 220409(R) (2019).

82) M. Beens, M. L. M. Lalieu, R. A. Duine, and B. Koopmans, AIP Adv. 9, 125133 (2019).

83) A. Baral and H. C. Schneider, Phys. Rev. B 91, 100402(R) (2015).

84) A. M. Kalashnikova and V. I. Kozub, Phys. Rev. B 93, 054424 (2016).

85) V. N. Gridnev, J. Phys.: Condens. Matter 28, 476007 (2016).

86) V. N. Gridnev, Phys. Rev. B 98, 014427 (2018).

87) V. N. Gridnev, Phys. Rev. B 100, 174405 (2019).

88) C. S. Davies, T. Janssen, J. H. Mentink, A. Tsukamoto, A. V. Kimel, A. F. G. Van Der Meer, A. Stupakiewicz, and A. Kirilyuk, Phys. Rev. Appl. 13, 024064 (2020).

89) V. G. Bar'yakhtar, Sov. Phys. JETP 60, 863 (1984).

90) D. Garanin, Phys. Rev. B 55, 3050 (1997).

91) S. Anisimov, B. Kapeliovich, and T. Perel'Man, Sov. J. Exp. Theor. Phys. 39, 776 (1974).

92) J. K. Chen, D. Y. Tzou, and J. E. Beraun, Int. J. Heat Mass Transf. 49, 307 (2006).

93) J. Pudell, A. A. Maznev, M. Herzog, M. Kronseder, C. H. Back, G. Malinowski, A. von Reppert, and M. Bargheer, Nat. Commun. 9, 3335 (2018).

94) J. E. Pudell, M. Mattern, M. Hehn, G. Malinowski, M. Herzog, and M. Bargheer, Adv. Funct. Mater. 30, 2004555 (2020).

95) R. B. Wilson, J. Gorchon, Y. Yang, C. H. Lambert, S. Salahuddin, and J. Bokor, Phys. Rev. B 95, 180409(R) (2017)

96) M. D. Stiles, J. Xiao, and A. Zangwill, Phys. Rev. B 69, 054408 (2004).

97) G. M. Choi, C. H. Moon, B. C. Min, K. J. Lee, and D. G. Cahill, Nat. Phys. 11, 576 (2015)

98) I. H. Shin, B. C. Min, B. K. Ju, and G. M. Choi, Jpn. J. Appl. Phys. 57, 090307 (2018).

99) T. S. Seifert, S. Jaiswal, J. Barker, S. T. Weber, I. Razdolski, J. Cramer, O. Gueckstock, S. F. Maehrlein, L. Nadvornik, S. Watanabe, C. Ciccarelli, A. Melnikov, G. Jakob, M. Münzenberg, S. T. B. Goennenwein, G. Woltersdorf, B. Rethfeld, P. W. Brouwer, M. Wolf, M. Kläui, and T. Kampfrath, Nat. Commun. 9, 2899 (2018)

100) I. Razdolski, A. Alekhin, N. Ilin, J. P. Meyburg, V. Roddatis, D. Diesing, U. Bovensiepen, and A. Melnikov, Nat. Commun. 8, 15007 (2017).

101) G. M. Choi and B. C. Min, Phys. Rev. B 97, 014410 (2018).

102) K. Sato and Y. Togami, J. Magn. Magn. Mater. 35, 181 (1983).

103) M. Beens, R. A. Duine, and B. Koopmans, Phys. Rev. B 102, 054442 (2020).

104) Ł. Cywiński and L. J. Sham, Phys. Rev. B 76, 045205 (2007).

105) E. G. Tveten, A. Brataas, and Y. Tserkovnyak, Phys. Rev. B 92 , 180412(R) (2015).

106) Y. Yang, R. B. Wilson, J. Gorchon, C. H. Lambert, S. Salahuddin, and J. Bokor, Sci. Adv. 3, e1603117 (2017).

107) D. O. Ignatyeva, C. S. Davies, D. A. Sylgacheva, A. Tsukamoto, H. Yoshikawa, P. O. Kapralov, A. Kirilyuk, V. I. Belotelov, and A. V. Kimel, Nat. Commun. 10, 4786 (2019).

108) A. Dutta, A. V. Kildishev, V. M. Shalaev, A. Boltasseva, and E. E. Marinero, Opt. Mater. Express 7, 4316 (2017).

109) D. Steil, S. Alebrand, A. Hassdenteufel, M. Cinchetti, and M. Aeschlimann, Phys. Rev. B 84, 224408 (2011).

110) H. Yoshikawa, S. El Moussaoui, S. Terashita, R. Ueda, and A. Tsukamoto, Jpn. J. Appl. Phys. 55, 07MD01 (2016).

111) H. Yoshikawa, S. Kogure, S. Toriumi, T. Sato, A. Tsukamoto, and A. Itoh, J. Magn. Soc. Jpn. 38, 139 (2014)

112) J. Y. Chen, L. He, J. P. Wang, and M. Li, Phys. Rev. Appl. 7, 021001 (2017).

113) A. El-Ghazaly, B. Tran, A. Ceballos, C. H. Lambert, A. Pattabi, S. Salahuddin, F. Hellman, and J. Bokor, Appl. Phys. Lett. 114, 232407 (2019). 


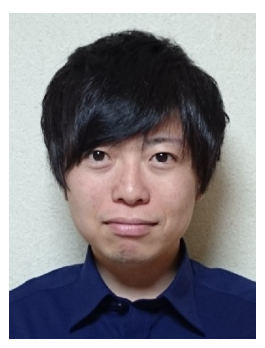

Satoshi Iihama was born in Nagaoka city in 1988. He received his Ph.D. degree in 2016 at Tohoku University. He then joined spintronics research center at the National Institute of Advanced Industrial Science and Technology as JSPS postdoctoral fellow (2016-2018). He joined Professor Stéphane Mangin's group at Institute Jean Lamour (Nancy, France) during postdoctoral fellow as a visiting researcher (2017-2018). He then joined Professor Shigemi Mizukami group at Advanced Institute for Materials Research, Tohoku University since 2018. He is currently Assistant Professor at Frontier Research Institute for Interdisciplinary Sciences, Tohoku University. His research topic is magnetization dynamics driven by femtosecond light pulses.

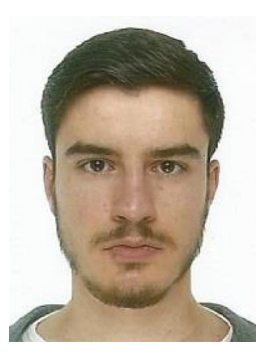

Quentin Remy was born in Epinal, France in 1994. He obtained a M.Sc. degree in Materials Engineering from Université de Lorraine, France (2018) and a M.Sc. in Chemical Engineering from University of Alberta, Canada (2018). He is currently finishing his $\mathrm{Ph} . \mathrm{D}$. at Institut Jean Lamour, France where he studies the ultrafast magnetization reversal of ferrimagnetic and ferromagnetic thin films.

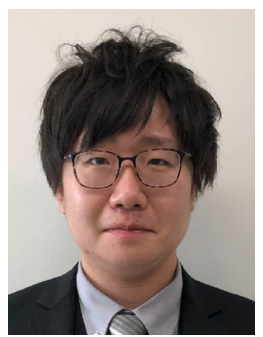

Junta Igarashi received the Ph.D. degree from Tohoku University, Sendai, Japan, in 2021. He was an intern at Institute Jean Lamour, Lorraine University, Nancy, France to work on all-optical switching in spin valve $(2018,2019)$. He is currently postdoctoral fellow at Research Institute of Electrical Communication, Tohoku University. He has been working on research and development of spintronics and devices.

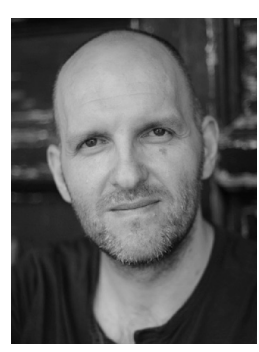

Grégory Malinowski obtained his Ph.D. degree in 2004 at the Université Henri Poincare Nancy, France. He then joined the group Magnetism and Spin electronics of Professor Coey at the Trinity College Dublin, Ireland (2005-2006) before to move to the Eindhoven University of Technology in the group Physics of Nanostructures of Professor Bert Koopmans, Netherlands (2007-2008). After a short postdoctoral fellowship in the group of Dr. Mathias Kläui at the University of Konstanz, Germany (2009), he obtained a CNRS researcher position in the group IDMAG at the Laboratoire de Physique des Solides in Orsay, France. Since 2013, he moved to the Jean Lamour Institute, Lorraine University in Nancy. His main research activity focuses on ultrafast magnetization dynamics and manipulation as well as ultrafast spin transport in magnetic multilayers.

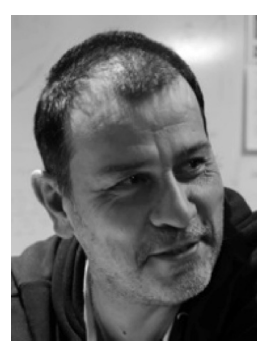

Michel Hehn received the Ph.D. degree from the University of Strasbourg in 1996, joined the French National Center of Scientific Research in 1998 and became professor at University de Lorraine in 2006. $\mathrm{He}$ is also a member of the IUF (Institut Universitaire de France). He is a specialist in material growth and in nanomagnetism/spintronics. In 2010, he won the Yves Rocard 2010 Price of the French Society of Physics for the invention and the technological development of "a new generation of magnetic sensors for ASB" for the SNR society. He has co-authored more than 270 papers in refereed international journals and in proceeding of international conferences.

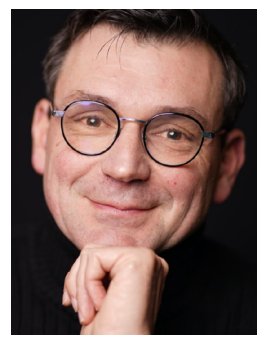

Stéphane Mangin was born in Nancy (France) in 1971. He received his Ph.D. degree in 1997 from Joseph Fourier university in Grenoble. After a Postdoc in KU-Leuven he became an assistant Professor at Lorraine University. In 2004-2005 he was a visiting Scientist at the IBM-Hitachi Research Center in San Jose (US) and in 2012-2013 he was visiting professor at UC-San Diego (US). He has work on magnetisation manipulation at the nanometer scale using various stimulus (charge and spin current, electric field, light etc.). He became full professor in 2007 and the head of the Spintronic and Nanomagnetism team at IJL in 2010. He is fellow of the American Physical Society and fellow of the IEEE magnetic society. 